\title{
SPECTROSCOPIC ORBITAL PERIODS FOR 29 CATACLYSMIC VARIABLES FROM THE SLOAN DIGITAL SKY SURVEY
}

\author{
John R. Thorstensen ${ }^{1}$, Cynthia J. Taylor ${ }^{1}$, Christopher S. Peters ${ }^{1}$, Julie N. Skinner ${ }^{1}$, John Southworth ${ }^{2}$, and \\ BORIS T. GäNSICKE ${ }^{3}$ \\ ${ }^{1}$ Department of Physics and Astronomy 6127 Wilder Laboratory, Dartmouth College Hanover, NH 03755-3528, USA \\ 2 Astrophysics Group Keele University Staffordshire ST5 5BG, UK \\ ${ }^{3}$ Department of Physics University of Warwick Coventry CV4 7AL, UK \\ Received 2014 August 29; accepted 2015 February 6; published 2015 March 12
}

\begin{abstract}
We report follow-up spectroscopy of 29 cataclysmic variables from the Sloan Digital Sky Survey (SDSS), 22 of which were discovered by SDSS and seven of which are previously known systems that were recovered in SDSS. The periods for 16 of these objects were included in the tabulation by Gänsicke et al. While most of the systems have periods less than $2 \mathrm{hr}$, only one has a period in the 80-86 minutes "spike" found by Gänsicke et al., and 11 have periods longer than $3 \mathrm{hr}$, indicating that the present sample is skewed toward longer-period, higher-luminosity objects. Seven of the objects have spectra resembling dwarf novae, but have apparently never been observed in outburst, suggesting that many cataclysmics with relatively low variability amplitude remain to be discovered. Some of the objects are notable. SDSS J07568+0858 and SDSS J08129+1911 were previously known to have deep eclipses; in addition to spectroscopy, we use archival data from the Catalina Real Time Transient Survey to refine their periods. We give a parallax-based distance of $195(+54,-39)$ pc for LV Cnc (SDSS J09197+0857), which at $P_{\text {orb }}=81 \mathrm{~m}$ has the shortest orbital period in our sample. SDSS J08091+3814 shows both the spectroscopic phase offset and phase-dependent absorption found in SW Sextantis stars. The average spectra of SDSS J08055+0720 and SDSS J16191+1351 show contributions from K-type secondaries, and SDSS J080440 +0239 shows a contribution from an early M star. We use these to constrain the distances. SDSS J09459+2922 has characteristics typical of a magnetic system. SDSS11324+6249 may be a novalike variable, and if so, its orbital period ( 99 minutes) is unusually short for that subclass.
\end{abstract}

Key words: stars: general

Supporting material: machine-readable and VO tables

\section{INTRODUCTION}

Cataclysmic variable stars (CVs) are close binary systems in which a white dwarf primary star accretes matter by way of Roche lobe overflow from a companion (the secondary), which usually resembles a late-type main-sequence $\operatorname{star}^{4}$ (Warner 1995). Because the red-giant precursor of a white dwarf can become much larger than the semimajor axis of a typical CV, it is thought that CVs are remnants of common-envelope evolution. Subsequent evolution proceeds through loss of orbital angular momentum, probably through magnetic braking of the co-rotating secondary, and at the shortest periods through gravitational radiation losses. For most systems, the loss of angular momentum causes the orbital period to decrease, but eventually the secondary becomes degenerate, so that subsequent mass loss causes its radius to increase. If this point has been passed, the orbital period should increase gradually as mass transfer continues. This evolutionary turnaround, known as the "period bounce," is predicted to occur at orbital period $P_{\text {orb }}$ near 65 minutes, although empirically the minimum period appears to be somewhat longer ( 75 minutes; Barker \& Kolb 2003). Standard evolutionary theory, then, predicts that few CVs will be found below the period minimum. Also, the gravitational radiation thought to dominate the angular momentum losses at short periods works rather slowly, so a relatively large number of $\mathrm{CVs}$ should have periods just

\footnotetext{
4 Some objects have white dwarf secondaries (as well as primaries); these are known as AM CVn stars, and while they are usually considered to be CVs, we are not including them in this discussion.
}

longward of the minimum, forming a "spike" in the distribution.

Until recently, observational evidence for this scenario was somewhat limited. CVs with hydrogen-rich secondaries do respect the lower period bound; the few exceptions appear to be either stars that evolved significantly before beginning mass transfer (Augusteijn et al. 1996; Thorstensen et al. 2002) or unusual objects of other provenance (Littlefair et al. 2007; Patterson et al. 2008). But the distribution of known CV periods did not show the predicted spike (Patterson 1998; Barker \& Kolb 2003; Gänsicke et al. 2009).

The mediocre agreement with theory prompts us to consider how CVs are selected. Until recently, CVs were detected mostly through photometric variability (especially dwarf nova outbursts), X-ray emission, and ultraviolet color. The Sloan Digital Sky Survey (SDSS) turned up a large number of new CVs (Szkody et al. 2002, 2003, 2004, 2005, 2006, 2007, 2009, hereafter collectively referred to as SzkodySDSSCVs). CV colors are unusual because of their composite spectra, with the result that SDSS allotted spectroscopic fibers to many of them. Although CV spectra are heterogeneous, they tend to be fairly distinctive, so many of the CVs could be classified with high confidence. The criteria for allotting spectroscopic fibers to stellar objects were mostly designed to generate a large sample of galaxies and QSOs, not to observe every available CV, so there is no assurance that the SDSS CV sample is truly complete. Nonetheless, the combination of the great depth of SDSS, and the lack of bias toward outbursting objects, led to the discovery of a large number of low-luminosity objects just 
Table 1

List of Objects

\begin{tabular}{|c|c|c|c|c|c|c|}
\hline Abbreviation & Name & $\begin{array}{c}\alpha_{2000} \\
(\mathrm{~h} \mathrm{~m} \mathrm{~s})\end{array}$ & $\begin{array}{l}\delta_{2000} \\
(0, \prime \prime)\end{array}$ & $\begin{array}{c}g \\
(\mathrm{mag})\end{array}$ & $\begin{array}{c}\text { Synth. } V \\
\text { (mag) }\end{array}$ & References \\
\hline SDSS0756 & $\ldots$ & 075653.11 & +08 5831.8 & 16.27 & 16.8 & VIII \\
\hline SDSS0805 & $\cdots$ & 080534.49 & +07 2029.1 & 18.52 & 18.5 & VI \\
\hline SDSS0809 & $\ldots$ & 080908.39 & +38 1406.2 & 15.61 & 15.7 & II \\
\hline SDSS0812 & $\cdots$ & 081256.85 & +191157.8 & 15.80 & 16.0 & $\mathrm{~V}$ \\
\hline SDSS0813 & $\ldots$ & $\begin{array}{lll}08 & 13 & 52.02\end{array}$ & +281317.3 & 17.16 & 17.4 & IV \\
\hline SDSS0838 & $\ldots$ & 083845.26 & +491055.1 & 19.59 & 18.5 & I \\
\hline SDSS0844 & V495 Hya & 084400.10 & +02 3919.3 & 18.34 & 18.7 & II \\
\hline SDSS0912 & $\ldots$ & $09 \quad 1242.18$ & +620940.1 & 18.81 & 19.4 & VIII \\
\hline SDSS0916 & $\mathrm{HH} \mathrm{Cnc}$ & 091650.78 & +284943.7 & 19.17 & 19.1 & VI \\
\hline SDSS0919 & LV Cnc & 091945.11 & +085710.0 & 18.20 & 18.8 & IV \\
\hline SDSS0922 & $\ldots$ & 092229.26 & +330743.6 & 18.44 & 19.3 & IV \\
\hline SDSS0945 & $\ldots$ & 094558.24 & +292253.2 & 19.10 & 19.1 & VI \\
\hline SDSS1013 & $\ldots$ & 101323.64 & +455858.9 & 18.86 & 19.3 & III \\
\hline SDSS1019 & $\mathrm{AC} \mathrm{LMi}^{\mathrm{a}}$ & 101947.26 & +335753.6 & 18.39 & 17.8 & VI \\
\hline SDSS1023 & NSV 04838 & 102320.28 & +440509.9 & 18.83 & 18.8 & IV \\
\hline SDSS1028 & Leo5 & 102800.09 & +214813.5 & 16.34 & 16.2 & VII \\
\hline SDSS1131 & MR UMa & 113122.39 & +432238.6 & 16.17 & 17.9 & $\mathrm{~V}$ \\
\hline SDSS1132 & $\ldots$ & 113215.50 & +624900.4 & 18.49 & 18.4 & III \\
\hline SDSS1244 & $\ldots$ & 124417.89 & +300401.0 & 18.61 & 19.1 & VII \\
\hline SDSS1429 & $\ldots$ & 142955.86 & +414516.8 & 17.70 & 18.3 & IV \\
\hline SDSS1504 & ${\text { Boo } 1^{b}}^{b}$ & 150441.76 & +084752.6 & 19.44 & 19.2 & VII \\
\hline SDSS1536 & $\ldots$ & 153634.42 & +332851.9 & 19.20 & 19.0 & IV \\
\hline SDSS1556 & V493 Ser & 155644.23 & -000950.3 & 18.05 & 18.7 & I \\
\hline SDSS1610 & V519 Ser & 161007.49 & +035233.2 & 17.36 & 17.8 & VII \\
\hline SDSS1619 & $\ldots$ & 161909.10 & +135145.5 & 18.49 & 17.2 & VII \\
\hline SDSS1653 & V1227 Her & 165359.06 & +201010.4 & 17.53 & 18.5 & $\mathrm{~V}$ \\
\hline SDSS1656 & V1229 Her & 165658.13 & +212139.3 & 18.52 & 18.9 & $\mathrm{~V}$ \\
\hline SDSS1659 & $\ldots$ & 165951.69 & +192745.6 & 16.76 & 17.3 & $\mathrm{~V}$ \\
\hline SDSS1730 & $\ldots$ & 173008.38 & +624754.7 & 15.92 & 17.8 & I \\
\hline
\end{tabular}

Notes. Coordinates and $g$ magnitudes are taken from the discovery papers referred to in the last column. Synthetic $V$ magnitudes (column 4$)$ are from our average spectra. References are as follows: I—Szkody et al. (2002), II—Szkody et al. (2003), III—Szkody et al. (2004), IV—Szkody et al. (2005), V—Szkody et al. (2006), VI-Szkody et al. (2007), VII—Szkody et al. (2009), VIII—Szkody et al. (2011).

a Also known as HS1016+3412 (Aungwerojwit et al. 2006).

${ }^{\mathrm{b}}$ As listed in Downes et al. (2001).

(This table is available in machine-readable and Virtual Observatory (VO) forms.)

above the period minimum. Gänsicke et al. (2009) compiled results for 137 SDSS CVs (most of them newly discovered), showed that their orbital period distribution was significantly different from previously known samples, and identified the short-period objects with the long-predicted period spike.

Here we present spectroscopy and orbital periods for 29 CVs; Table 1 lists the objects and gives the short names we will use for them here. Twenty-two of these were originally discovered in SDSS data. We also include seven more objects that had been known previously but were recovered in the SDSS. Gänsicke et al. (2009) listed orbital periods for 16 of the objects, and we give the details here. Observations of the remaining objects were not completed until after the Gänsicke et al. (2009) paper was prepared. The periods for most of these appear here for the first time.

\section{OBSERVATIONS AND REDUCTIONS}

\subsection{MDM Observations and Reductions}

Most of the observations reported here are from the $2.4 \mathrm{~m}$ Hiltner telescope at MDM Observatory on Kitt Peak, Arizona, equipped with the modular spectrograph, a 600 line $\mathrm{mm}^{-1}$ grating, and a $2048^{2}$ SITe CCD (the "Echelle" detector). This gave $2.0 \AA \mathrm{pixel}^{-1}$ and a spectral resolution of $\sim 3.5 \AA$ from
4210 to $7500 \AA$, with strong vignetting toward the ends of the spectral range. For some of the later observations, we used a $1024^{2}$ SITe CCD ("Templeton"), which had the same pixel as "Echelle" and scale and similar sensitivity, but less spectral coverage (4660-6730 $)$. The projected slit width was $\sim 1.1$ arcsec. We often observed far from the meridian to resolve ambiguities in the daily cycle count; when doing this, we rotated the instrument to position the slit near the parallactic angle, to minimize atmospheric dispersion effects Filippenko (1982)..$^{5}$ During twilight we took spectra of bright early-type stars to derive an approximate correction for the telluric bands, flux standard stars when conditions warranted, and $\mathrm{He}, \mathrm{Ne}$, and Xe lamps for wavelength calibration. For spectra taken during the night, we used the $\lambda 5577$ night sky line to establish the velocity zero point. The night-sky line technique lets us monitor the radial velocities as they come in, making for very efficient use of observing time. Individual exposures were typically 6- 8 minutes, to avoid smearing the velocities excessively at short periods.

\footnotetext{
5 The most recent version of JRT's JSkyCalc observing planning program includes a tool for computing the optimum position angle for a spectrograph slit over an observation interval; it is available at http:// dartmouth.edu/ physics/labs/skycalc/flyer.html.
} 
Two of the objects, SDSS0812 and SDSS1028 (Leo5), were bright enough for the McGraw-Hill $1.3 \mathrm{~m}$ telescope, also at MDM. For this we used the Mark III spectrograph, the SITe $1024^{2}$ CCD "Templeton," and a grism giving $2.2 \AA \mathrm{pixel}^{-1}$ from 4650 to $6980 \AA$, with a spectral resolution of $4.0 \AA$.

Whenever the sky was clear, we observed flux standard stars in twilight. We estimate that the absolute fluxes are accurate to about $20 \%$, because of transparency variations and uncalibratable losses at the 1.1 arcsec slit. In addition, the modspec spectra sometimes suffer from irreproducible, wavelengthdependent variations in the response, which can reach 20 or $30 \%$ amplitude in individual spectra. For the most part these average out fairly well when large numbers of spectra are combined. The $V$ magnitudes given in Table 1 were synthesized from our flux-calibrated spectra using the iraf sbands task and the $V$ passband tabulated by Bessell (1990).

Our average spectra are included in Figures 1 through 8 (along with the period searches and folded velocity curves discussed later). The line strengths, line profiles, continuum shapes, and flux levels are mostly similar to those in SzkodySDSSCVs. The $V$ magnitudes inferred from our spectral continua (Table 1) also agree fairly well with the $g$ magnitudes from SDSS imaging. We therefore observed most of the systems in states similar to when they were discovered; exceptions are noted in the discussions of individual objects (Section 3).

We reduced the MDM data primarily with IRAF routines, but for extracting one-dimensional spectra from the twodimensional images, we used our own implementation of the optimal-extraction algorithm described by Horne (1986).

To measure radial velocities of the $\mathrm{H} \alpha$ emission line, we used an algorithm developed by Schneider \& Young (1980), in which the line profile is convolved with an antisymmetric function and the zero of the convolution is taken to be the line center. The antisymmetric function was usually composed of positive and negative Gaussians, separated by an interval chosen to emphasize the steep parts of the line profile. Uncertainties in the velocities were estimated by propagating the errors of the counts in each spectral channel, as estimated from photon statistics and read noise; line-profile variations can introduce scatter significantly larger than this. For absorptionline velocities we used the rvsao cross-correlation package (Kurtz \& Mink 1998); the template was the sum of a large number of late-type IAU velocity standards shifted to zero velocity. The rvsao package estimates velocity uncertainties using the antisymmetric part of the cross-correlation (Tonry \& Davis 1979). Table 2 lists all the radial velocities.

To search for periods, we used the "residual-gram" algorithm described by Thorstensen et al. (1996). We fitted the time series with sinusoids at the best periods (see Thorstensen \& Freed 1985 for details); Table 3 lists the parameters of the curves that are plotted in Figures 1-8. Because essentially all of our observations are from a single longitude, the times of observation fall in a limited window modulo one sidereal day, so period searches inevitably show aliases spaced by 1 cycle day $^{-1}$, corresponding to alternate choices of daily cycle count. We minimized the resulting ambiguities by taking observations over as wide a range of hour angles as possible. When appropriate, we used the Monte Carlo test described by Thorstensen \& Freed (1985) to estimate the reliability of our choice of alias.

\subsection{WHT Observations and Reductions}

A few of our spectra are from the ISIS Double Beam Spectrograph at the William Herschel Telescope at La Palma. The instrument configuration, observation procedure, and reductions were essentially as described by Southworth et al. (2007), except that we implemented the Schneider \& Young (1980) radial-velocity measurements using a python-language program based on PyFits and NumPy.

\subsection{Distances from Secondary Stars}

The spectra of CVs sometimes show a contribution from a late type star, which can constrain the distance. To do this, we use an interactive program to scale and subtract spectra of stars of known spectral type from the program star's spectrum, and adjust the spectral type and scale factor to cancel the late-type features. The subtracted spectra are either M- and K-dwarfs classified respectively by Boeshaar (1976) or Keenan \& McNeil (1989), and taken with the same instrument as the program star, or SDSS M-dwarf templates from Bochanski et al. (2007). For each spectral type giving reasonable cancellation, we estimate the acceptable range of scale factors, and hence the apparent $V$ magnitudes of the secondary. We use the spectral type to find the surface brightness in the $V$-band, using data tabulated by Beuermann et al. (1999).

The orbital period, together with the assumption that the secondary must fill its Roche critical lobe, strongly constrains the secondary's radius $R_{2}$ : at a given $P_{\text {orb }}, R_{2}$ is roughly proportional to $M_{2}^{1 / 3}$, where $M_{2}$ is the secondary's mass. We seldom can measure $M_{2}$ directly, but using evolutionary scenarios (Baraffe \& Kolb 2000) and the known $P_{\text {orb, we }}$ constrain $M_{2}$ to a broad range of plausible values, which results in a relatively narrow range of radii thanks to the weak dependence. Combining $R_{2}$ with the surface brightness and radius gives an absolute $V$ magnitude. Comparing this with the apparent $V$ of the secondary's contribution, and including a correction for interstellar extinction (guided by Schlegel et al. 1998) gives an estimate of the distance. Note that we do not assume that the secondary is a main-sequence star.

Because spectral features tend to grow stronger toward later spectral types, there is often a correlation between the secondary's spectral type and the fraction of the light attributed to it. We therefore compute the distance and its uncertainty using a Monte Carlo approach, that is, by running the calculation many times, every time selecting each input value randomly from its possible range. Our quoted distance is the median of the resulting histogram, and the range that includes $68 \%$ of the results is our quoted "1-sigma" uncertainty. When picking input values, we select a spectral type and then pick a magnitude based on the allowed range at that spectral type. This explicitly accounts for the correlation.

\section{THE INDIVIDUAL STARS}

\subsection{SDSS J07568+0858}

Szkody et al. (2009) noted that the SDSS spectrum of this object shows strong He II $\lambda 4686$ emission, and suggested it may be an intermediate polar (IP, i.e., a magnetic system with a white dwarf spin period substantially shorter than $\left.P_{\text {orb }}\right)$. They also found a preliminary period near $2 \mathrm{hr}$. While the present paper was in preparation, Tovmassian et al. (2014) presented a comprehensive photometric and spectroscopic study of the 

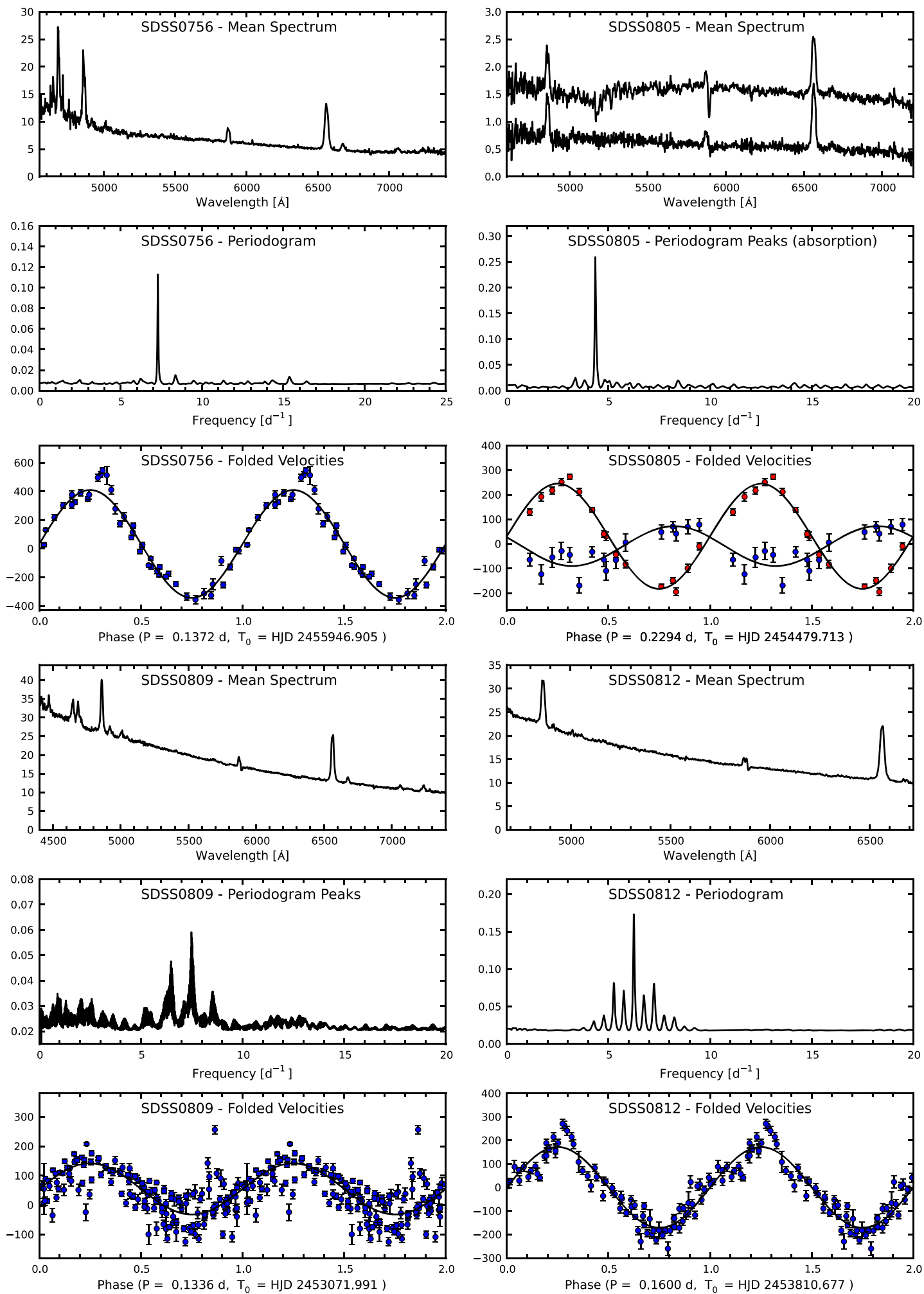

Figure 1. Average spectra, periodograms, and folded velocity curves for SDSS0756, SDSS0805, SDSS0809, and SDSS0812. The vertical scales, unlabeled to save space, are (1) for the spectra, $f_{\lambda}$ in units of $10^{-16} \mathrm{erg} \mathrm{s}^{-1} \mathrm{~cm}^{-2} \AA^{-1}$; (2) for the periodograms, $1 / \chi^{2}$ (dimensionless); and (3) for the radial velocity curves, barycentric radial velocity in $\mathrm{km} \mathrm{s}^{-1}$. For SDSS0805, the lower trace shows the remainder after subtracting the scaled spectrum of a K4 star (see text). In cases where velocities are from more than one observing run, the periodogram is labeled with the word "peaks," because the curve shown formed by joining local maxima in the full periodogram with straight lines. This suppresses fine-scale ringing due to the unknown number of cycle counts between runs. The folded velocity curves all show the same data plotted over two cycles for continuity, and the best-fit sinusoid (see 3) is also plotted. The velocities shown are H $\alpha$ emission velocities, but in SDSS0805 cross-correlation velocities of the secondary star are also plotted; they are shown in red in the online version, and are distinguished by their smaller error bars and larger velocity amplitude. 
Table 2

Radial Velocities

\begin{tabular}{|c|c|c|c|c|c|}
\hline $\begin{array}{l}\text { Star } \\
\text { SDSS J }\end{array}$ & Time $^{\mathrm{a}}$ & $\begin{array}{c}v_{\mathrm{emn}} \\
\left(\mathrm{km} \mathrm{s}^{-1}\right)\end{array}$ & $\begin{array}{c}\sigma \\
\left(\mathrm{km} \mathrm{s}^{-1}\right)\end{array}$ & $\begin{array}{c}v_{\mathrm{abs}} \\
\left(\mathrm{km} \mathrm{s}^{-1}\right)\end{array}$ & $\begin{array}{c}\sigma \\
\left(\mathrm{km} \mathrm{s}^{-1}\right)\end{array}$ \\
\hline $075653.11+085831.8$ & 2455944.9631 & -327 & 24 & $\ldots$ & $\ldots$ \\
\hline $075653.11+085831.8$ & 2455944.9713 & -253 & 20 & $\ldots$ & $\cdots$ \\
\hline $075653.11+085831.8$ & 2455944.9886 & 131 & 11 & $\cdots$ & $\ldots$ \\
\hline $075653.11+085831.8$ & 2455945.0084 & 324 & 16 & $\cdots$ & $\cdots$ \\
\hline $075653.11+085831.8$ & 2455945.0170 & 347 & 16 & $\cdots$ & $\cdots$ \\
\hline $075653.11+085831.8$ & 2455945.7396 & 13 & 17 & $\cdots$ & $\ldots$ \\
\hline
\end{tabular}

${ }^{a}$ Barycentric Julian Date of mid-exposure. The time base is UTC.

(This table is available in its entirety in machine-readable and Virtual Observatory (VO) forms.)

object, finding it to be an eclipsing system with $P_{\text {orb }}=0.1369745(4)$ days.

Our findings largely confirm those of Tovmassian et al. (2014). Our $\mathrm{H} \alpha$ velocities give an unambiguous $P_{\text {orb }}=3.292$ $\pm 0.003 \mathrm{hr}$, which is consistent with the much more precise value they find, and a velocity amplitude identical to theirs. By coincidence, the eclipse epoch given in their ephemeris (HJD 2455958.59184) is less than two weeks from the epoch of our spectroscopic observations, so we can compute eclipse-based phases for our velocities without significant uncertainty. In Figure 1 we show the velocities folded on the eclipse ephemeris. There is an intriguing indication of a rotational disturbance, i.e., a displacement toward positive velocity before eclipse, and a negative displacement following eclipse, as expected from the eclipse of a rotating structure.

The Catalina Real Time Transient Survey, Data Release 2 (Drake et al. 2009, hereafter CRTTS) ${ }^{6}$ gives 308 measurements of this source from 2005 to 2013 . The source is mostly around $16.3 \mathrm{mag}$, but with occasional excursions down to fainter than 18th-similar to the eclipse depth seen by Tovmassian et al. (2014). To see whether the fainter points were consistent with the eclipse, we downloaded the CRTTS data, corrected the timings to the solar system barycenter, and searched for periodicities near the known eclipse period using a "string length" algorithm (Dworetsky 1983). The Tovmassian et al. (2014) period is strongly detected, along with weak aliases corresponding to the annual cycle count.

The long time span of the CRTTS data allowed us to refine the period. The isolated observations from CRTTS do not permit the extraction of individual eclipse timings. To constrain the period, we instead varied the period slightly and examined the effect on folded light curves. As one might expect, the eclipse appeared sharply defined only for a narrow range of periods, namely $0.1369739(2)$ days. The central value is only 1.5 standard deviations from the Tovmassian et al. (2014) period, which is 0.1369745 (4) days. Figure 9 shows the CRTTS magnitudes folded using the refined period.

\subsection{SDSS J08055+0720}

The spectrum of this object shows a strong contribution from a secondary star along with emission lines and a continuum

\footnotetext{
6 The Catalina photometric data are available at http://nesssi.cacr.caltech.edu/ DataRelease/.
}

typical of dwarf novae at minimum light. However, the CRTTS shows the source near 18th magnitude without any outbursts detected despite good coverage over eight observing seasons.

We estimate the secondary's spectral type (see Section 2.3) to be $\mathrm{K} 4 \pm 1.5$ subtypes, and that it contributes a flux corresponding to $V=19.0 \pm 0.2 \mathrm{mag}$ (see Figure 1 ). The secondary's absorption-line velocities give a clean determination of the orbital period, which is $5.51 \mathrm{hr}$; the $\mathrm{H} \alpha$ emission velocities are not nearly as well behaved, but corroborate the absorption-line period. We have 16 velocities from 2008 January, and only a single point from 2008 March, which happened to fall near maximum velocity. The orbital frequencies that fit all the data are given approximately by $1 / P_{\text {orb }}=4.353 \pm 0.021 N$ cycle day ${ }^{-1}$, where $N$ is an integer and $-2 \leqslant N \leqslant+2$.

The secondary star is a little warmer than typically found in systems in this period range (Knigge 2006); given this, the evolutionary scenarios computed by Baraffe \& Kolb (2000) suggest that its mass is $0.75 \pm 0.2 M_{\odot}$. Schlegel et al. (1998) give a total reddening $E(B-V)=0.03$ for this celestial location $(l=214.76, b=19.81)$, so the extinction correction is small. The Monte Carlo procedure described in Section 2.3 gives $2500 \pm 400 \mathrm{pc}$ for the distance; at $2500 \mathrm{pc}$, the system would be $850 \mathrm{pc}$ from the Galactic plane.

The emission-line velocities are so noisy that they do not tell us much about the dynamics of the system, but the absorption velocities should trace the secondary's motion with reasonable fidelity. The velocity semi-amplitude, $K=216 \pm$ $12 \mathrm{~km} \mathrm{~s}^{-1}$, is fairly large for this orbital period. In addition, as noted above, the secondary's relatively early spectral type may indicate that it has not lost much mass. Taken together, the $K$-velocity and secondary mass estimate suggest that the white dwarf is on the massive side, and that the orbital inclination is not too far from edge-on. As an illustration, with $K=216 \mathrm{~km} \mathrm{~s}^{-1}$ and assuming $M_{2}=0.75 M_{\odot}$, the system would have $i=90^{\circ}$ (edge-on) with $M_{1}=0.85 M_{\odot}$; lower inclinations with these parameters would require still higher white-dwarf masses.

Our orbital period is corroborated by Woudt et al. (2012), who find ellipsoidal variations implying $P_{\text {orb }}=0.2287(5)$ days. While they do not detect eclipses, the ellipsoidal variation also suggests a fairly high orbital inclination. 
Table 3

Fits to Radial Velocities

\begin{tabular}{|c|c|c|c|c|c|c|}
\hline Data set & $T_{0}{ }^{\mathrm{a}}$ & $\begin{array}{c}P \\
(\mathrm{~d})\end{array}$ & $\begin{array}{c}K \\
\left(\mathrm{~km} \mathrm{~s}^{-1}\right)\end{array}$ & $\begin{array}{c}\gamma \\
\left(\mathrm{km} \mathrm{s}^{-1}\right)\end{array}$ & $N$ & $\begin{array}{c}\sigma^{\mathrm{b}} \\
\left(\mathrm{km} \mathrm{s}^{-1}\right)\end{array}$ \\
\hline SDSS0756 & $55946.9049(8)$ & $0.13718(11)^{\mathrm{c}}$ & $376(20)$ & $33(12)$ & 43 & 56 \\
\hline SDSS0805 abs. & $54479.713(2)$ & $0.2296(6)$ & $216(12)$ & $32(9)$ & 16 & 27 \\
\hline combined & $\ldots$ & $0.2294(6)$ & $\ldots$ & $\ldots$ & $\ldots$ & $\ldots$ \\
\hline SDSS0809 & $53071.991(3)$ & $0.133616(2)$ & $87(14)$ & $55(10)$ & 158 & 44 \\
\hline SDSS0812 & $53810.6770(20)$ & $0.1600(2)^{\mathrm{d}}$ & $172(13)$ & $-0(9)$ & 78 & 42 \\
\hline SDSS0844 & $53744.033(4)$ & $0.2070(7)$ & $47(6)$ & $54(4)$ & 89 & 20 \\
\hline SDSS0912 & $56299.0564(17)$ & $0.08013(9)$ & $101(15)$ & $-18(10)$ & 46 & 34 \\
\hline SDSS0916 & $56353.697(3)$ & $0.1845(5)$ & $31(3)$ & $8(2)$ & 43 & 7 \\
\hline SDSS0919 & $53746.7419(13)$ & $0.05647(12)$ & $65(9)$ & $73(6)$ & 64 & 29 \\
\hline SDSS0922 [WHT] & $54186.3557(12)$ & $0.06578(11)$ & $51(6)$ & $76(4)$ & 32 & 19 \\
\hline SDSS1019 & $54125.994(2)$ & $0.07915(14)$ & $45(9)$ & $19(6)$ & 31 & 20 \\
\hline SDSS1023 & $52672.773(2)$ & $0.0679(3)$ & $67(12)$ & $-86(9)$ & 42 & 29 \\
\hline SDSS1028 & $51191.860(4)$ & $0.14606(17)$ & $82(12)$ & $-75(9)$ & 74 & 32 \\
\hline SDSS1131 & $52671.8142(15)$ & $0.06325(10)$ & $37(6)$ & $-21(4)$ & 53 & 13 \\
\hline SDSS1132 & $54525.671(2)$ & $0.0689(2)$ & $28(5)$ & $-16(4)$ & 73 & 17 \\
\hline SDSS1244 & $54898.7621(17)$ & $0.07744(9)$ & $80(11)$ & $-8(8)$ & 59 & 25 \\
\hline SDSS1429 & $53549.677(3)$ & $0.0685(2):^{\mathrm{e}}$ & $86.0(234)$ & $-41(17)$ & 55 & 55 \\
\hline SDSS1504 & $55281.0322(19)$ & $0.08089(18)$ & $35(6)$ & $19(4)$ & 43 & 13 \\
\hline SDSS1536 & $56451.675(2)$ & $0.0921(2)$ & $88(12)$ & $-107(8)$ & 34 & 34 \\
\hline SDSS1556 & $52812.7011(15)$ & $0.08012(8)$ & $72(8)$ & $-31(6)$ & 52 & 26 \\
\hline SDSS1610 & $52328.0203(16)$ & $0.1322739(2)$ & $90(6)$ & $17(5)$ & 93 & 21 \\
\hline SDSS1619 abs. & $56453.839(3)$ & $0.2867(6)$ & $129(10)$ & $79(7)$ & 17 & 25 \\
\hline
\end{tabular}

Notes. Parameters of least-squares sinusoid fits to the radial velocities, of the form $v(t)=\gamma+K \sin \left(2 \pi\left(t-T_{0}\right) / P\right.$.

${ }^{a}$ Heliocentric Julian Date minus 2400000 . The epoch is chosen to be near the center of the time interval covered by the data, and within one cycle of an actual observation.

${ }^{\mathrm{b}}$ rms residual of the fit.

${ }^{\mathrm{c}}$ The eclipse period, refined from CRTTS data, is 0.1369739(2) days (see text).

d The eclipse period, refined from CRTTS data, is 0.1600525 (3) days (see text).

e The daily cycle count for SDSS1429 is ambiguous; see text for details.

\subsection{SDSS J08091+3814 = HS $0805+3822$}

This novalike variable was discovered in the Hamburg Quasar survey, and rediscovered in the SDSS. Rodríguez-Gil et al. (2007) found apparent grazing eclipses; their complete photometric data set was best fit with $P_{\text {orb }}=3.22 \mathrm{hr}$, but the eclipses alone could not rule out a period near $3.04 \mathrm{hr}$; Gänsicke et al. (2009) cite the $3.22 \mathrm{hr}$ period. Linnell et al. (2007) modeled the system using ultraviolet and optical spectra.

Our spectra come from four observing runs, but all appear similar, with a strong blue continuum and Balmer, $\mathrm{He}$ I, and He II emission lines.

Our best radial-velocity period, near $3.2068 \mathrm{hr}$, agrees roughly with the period adopted by Rodríguez-Gil et al. (2007) and Gänsicke et al. (2009), but disagrees on a finer scale. Rodríguez-Gil et al. (2007) find 0.1340385 (84) days, or $3.2169(2) \mathrm{hr}$, based on a count of 313 cycles in between their first and second grazing eclipse (their Table 1), but our radial velocities are not fit well at that period. However, an alternate fit assuming 314 cycles between their first two eclipses gives a best-fit period near $3.2069 \mathrm{hr}$, in excellent agreement with the best choice of spectroscopic alias. The 314-cycle eclipse fit is not quite as good-while their first two eclipses fit almost perfectly, their last two miss by -274 and +267 s respectively -but their best fit already shows deviations nearly this large, so the grazing eclipse evidently jitters somewhat in phase. Remarkably, although the radial velocity periodogram is riddled with aliases generated by different choices of cycle count between observing runs, the single best-fitting alias is the only radial-velocity alias that is compatible with the 314-cycle eclipse ephemeris. Because of this concordance, we are 

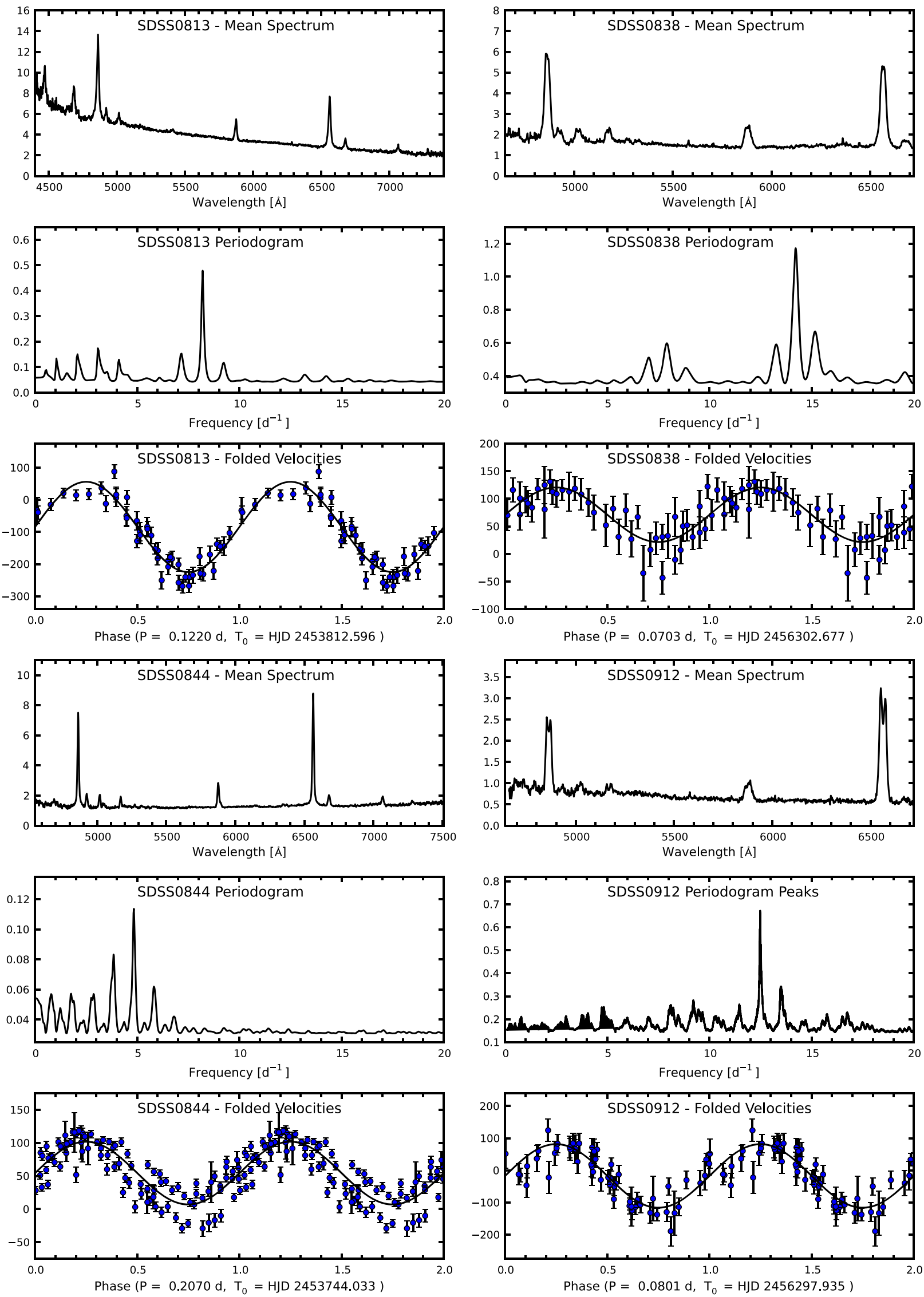

Figure 2. Similar to Figure 1, but for SDSS0813, SDSS0838, SDSS0844, and SDSS0912. 

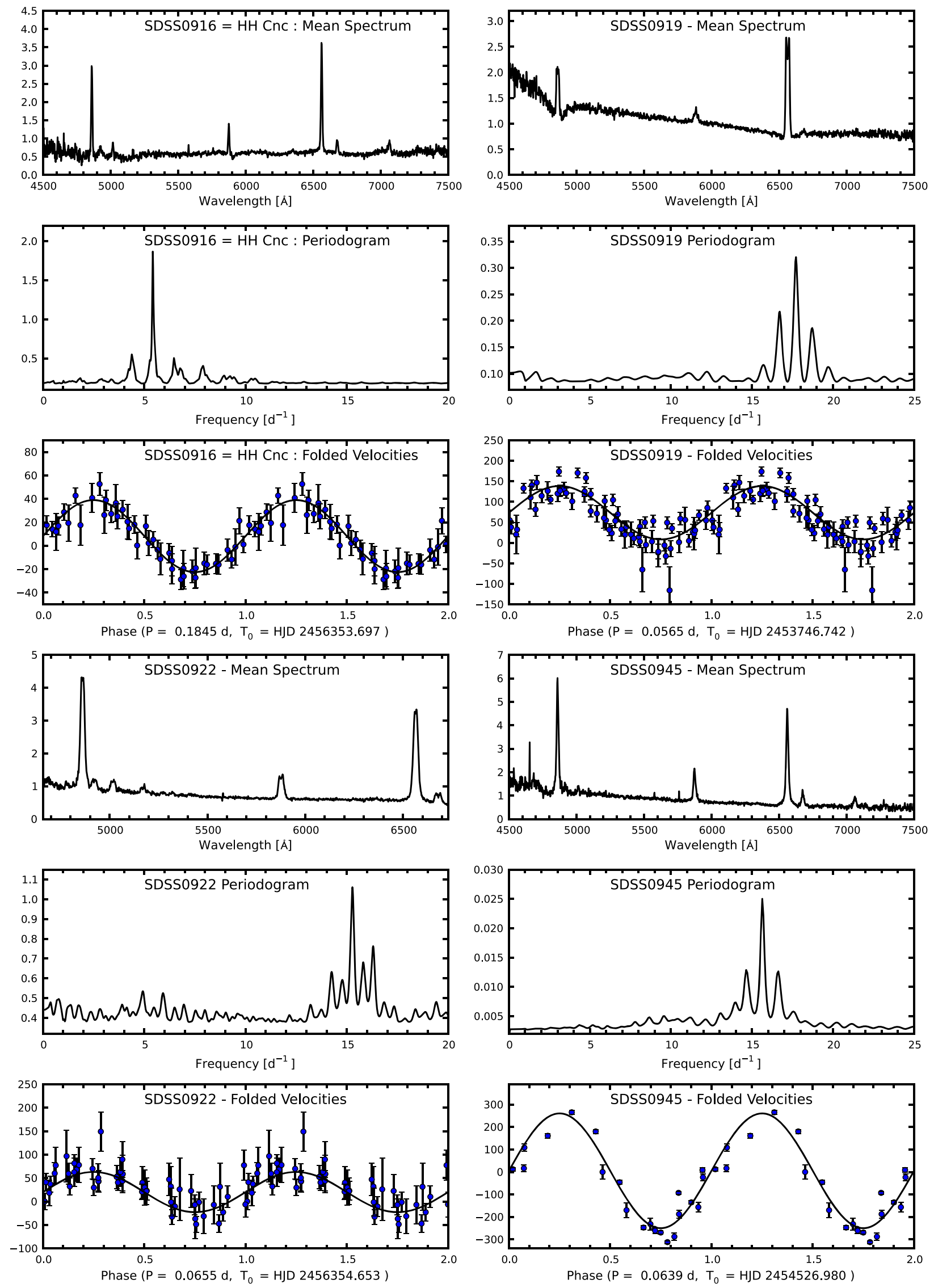

Figure 3. Similar to Figure 1, but for SDSS0916 = HH Cnc, SDSS0919, SDSS0922, and SDSS0945. 

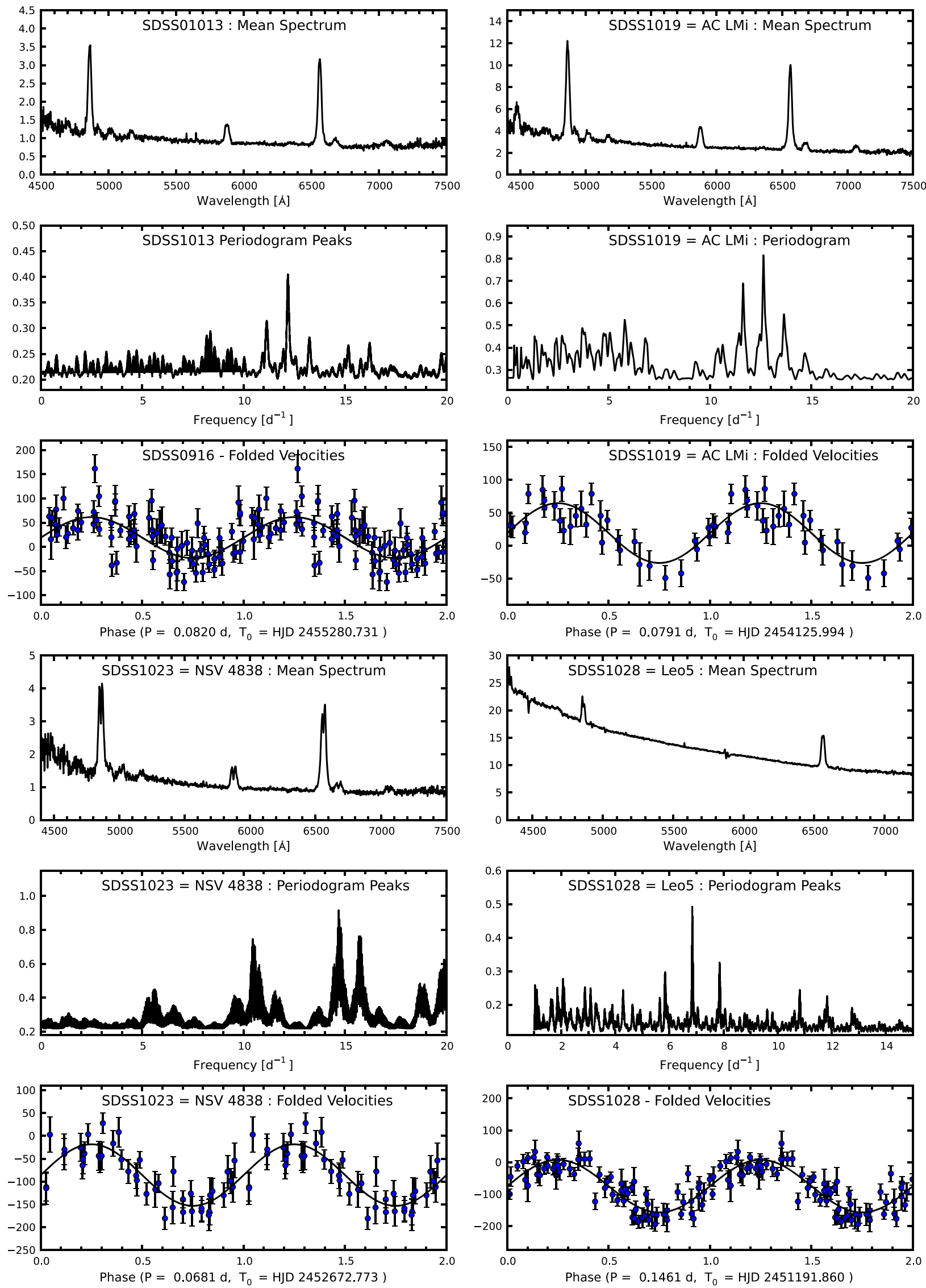

Figure 4. Similar to Figure 1, but for SDSS1013, SDSS1019 = AC LMi, SDSS1023 = NSV 4838, and SDSS1028 = Leo5. 

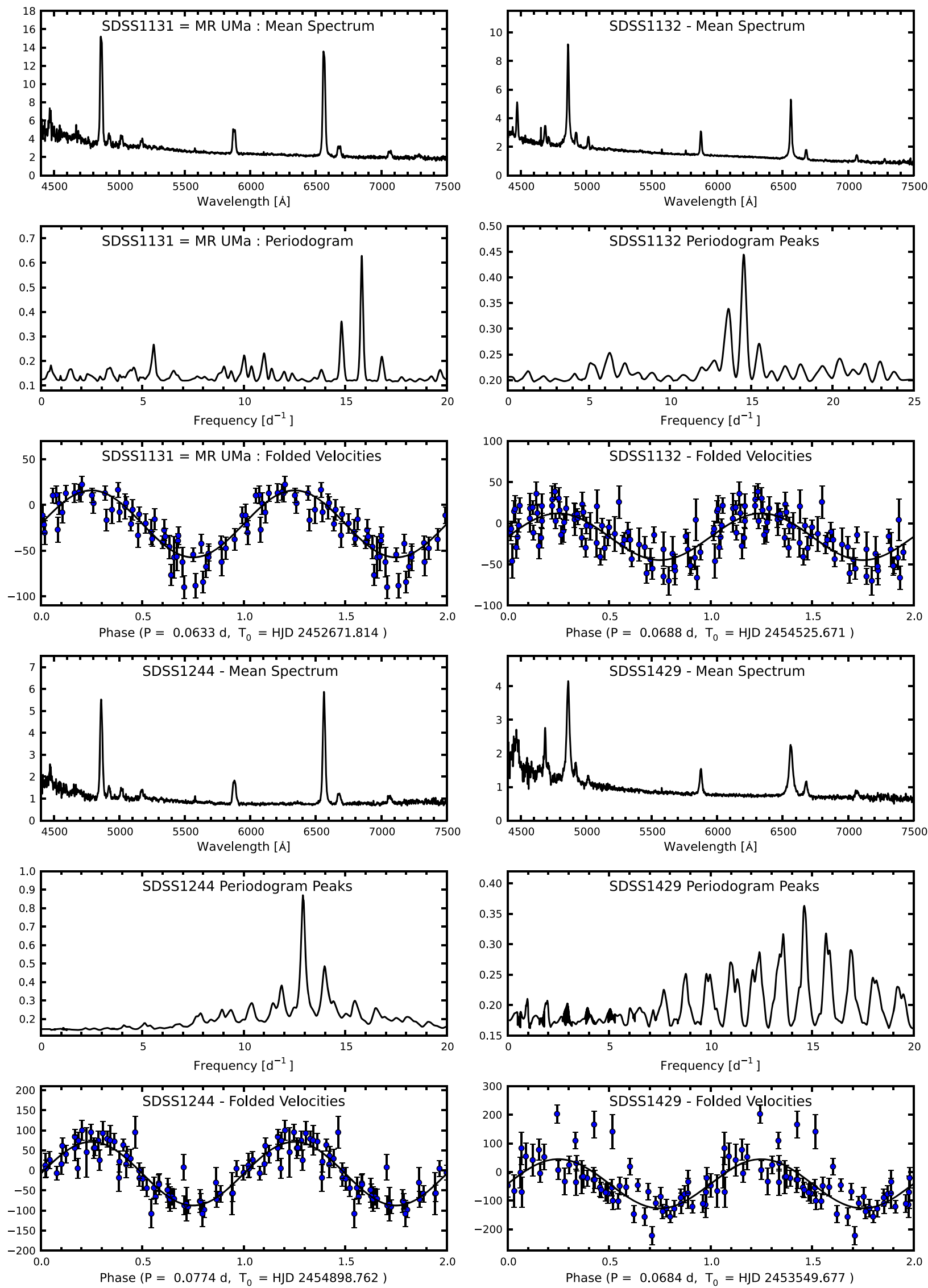

Figure 5. Similar to Figure 1, but for SDSS1131 = MR UMa, SDSS1132, SDSS1244, and SDSS1429. 

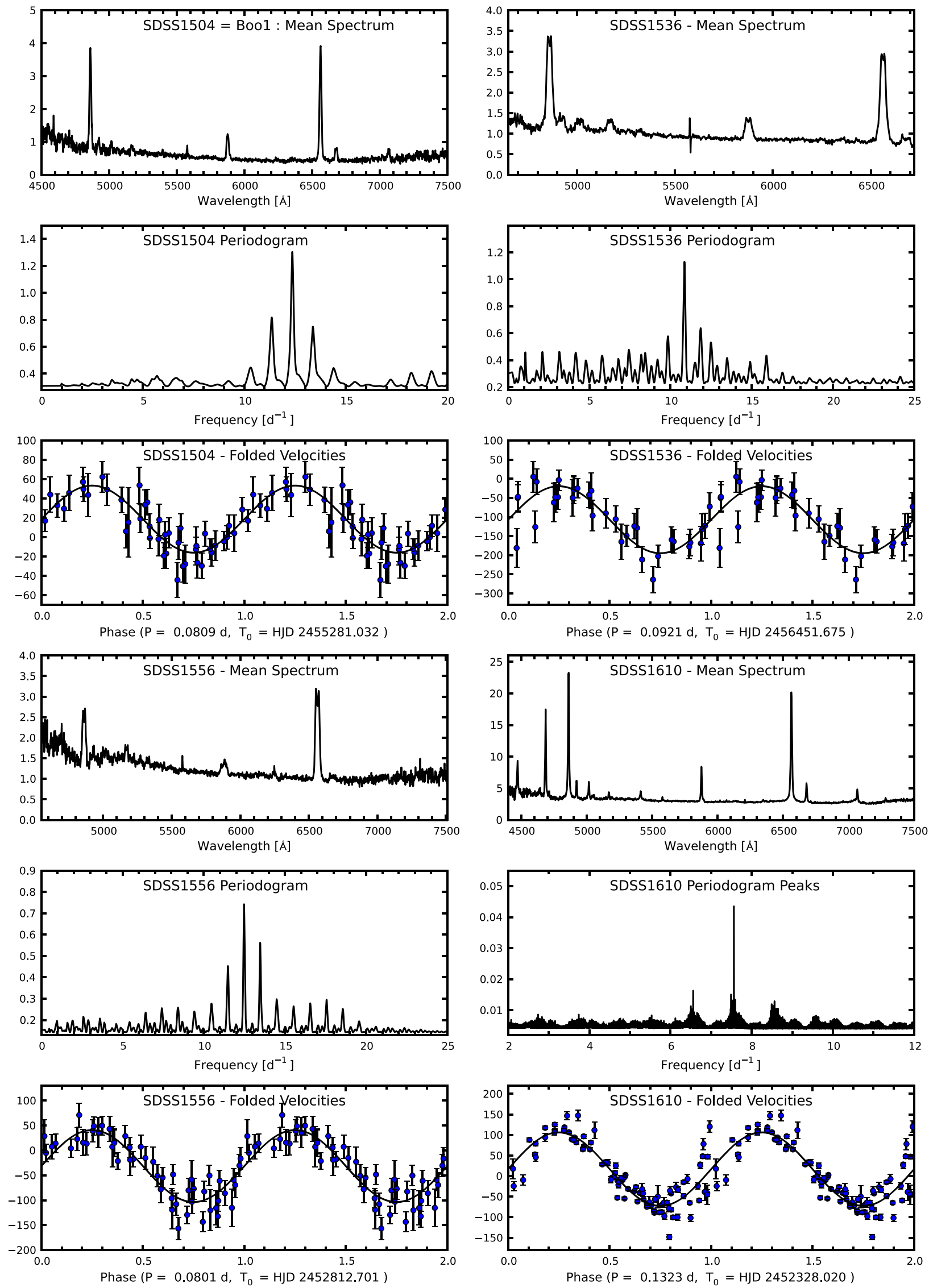

Figure 6. Similar to Figure 1, but for SDSS1504 = Boo1, SDSS1536, SDSS1556, and SDSS1610. 

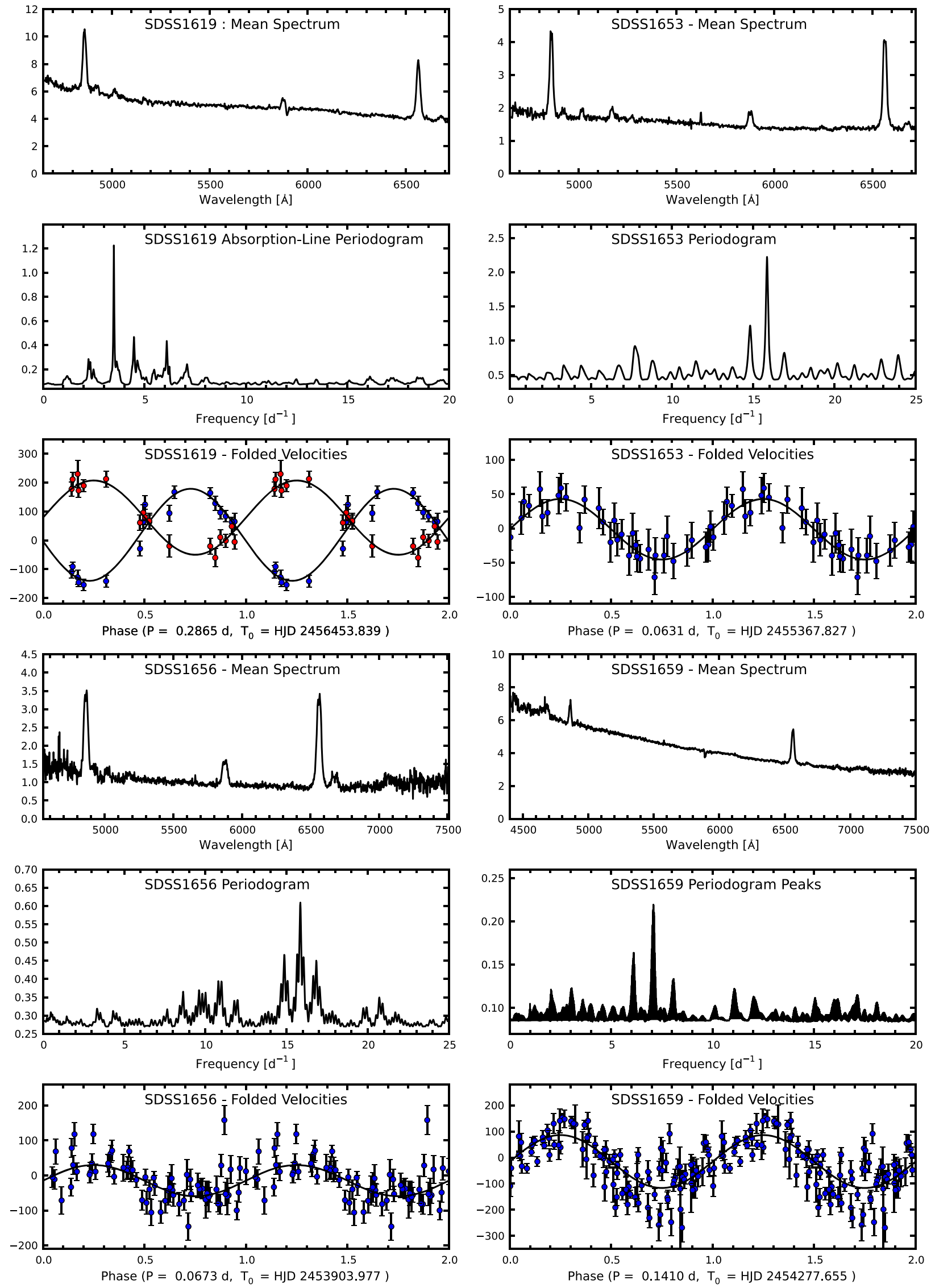

Figure 7. Similar to Figure 1, but for SDSS1619, SDSS1653, SDSS1656, and SDSS1659. For SDSS1619, both emission and absorption velocities are plotted. 

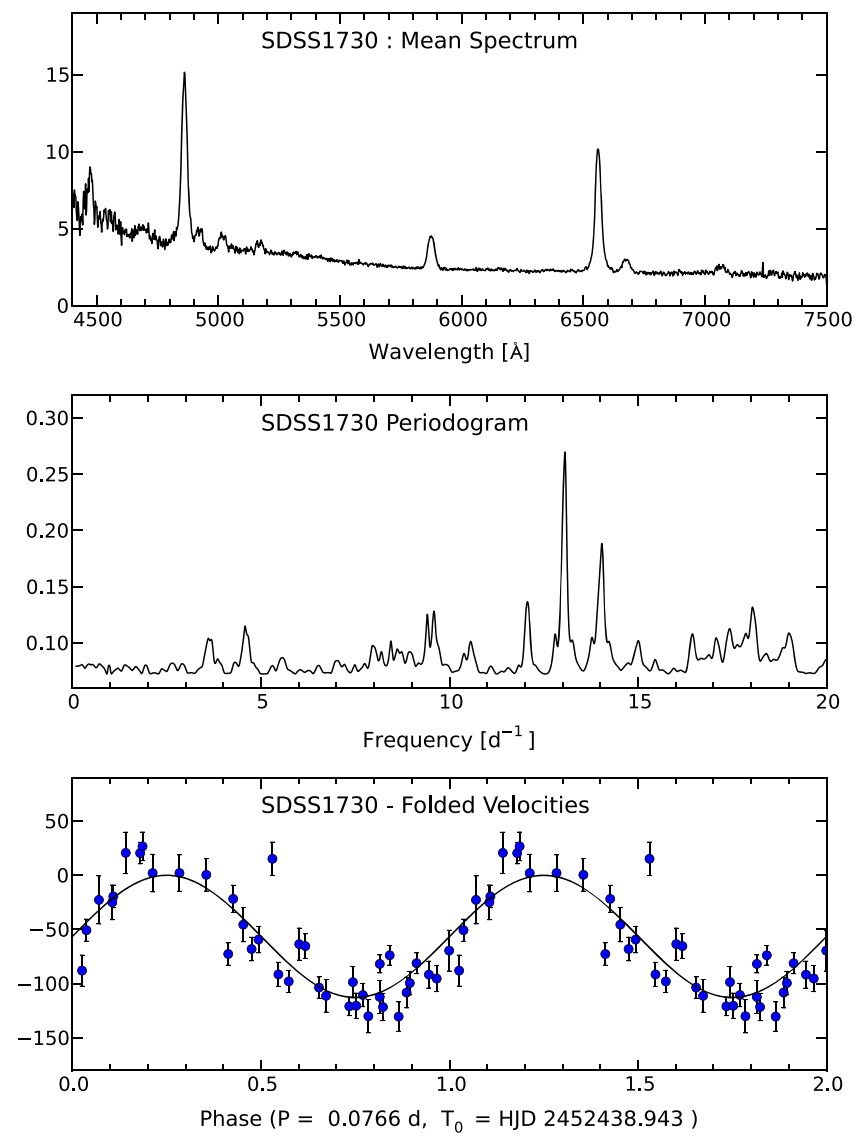

Figure 8. Similar to Figure 1, but for SDSS1730.

reasonably sure we have chosen the long-term velocity correctly, and quote the period in Table 3 with a correspondingly small uncertainty.

Rodríguez-Gil et al. (2007) include this object in a sample of SW Sex stars. In eclipsing SW Sex stars, the emission-line radial velocity curves and eclipses are offset in phase, in the sense that the eclipse occurs somewhat earlier than one would expect if the emission lines traced the white dwarf's motion. Fortunately, our radial velocities and the eclipses observed by Rodríguez-Gil et al. (2007) were obtained fairly close together in time-close enough so that we can compare their phases. The zero point of their eclipse ephemeris (their Equation (5)) occurs 2555.348(44) cycles after our radial-velocity epoch (Table 3). The radial-velocity is the red-to-blue crossing of the emission lines, so if the emission lines traced the white dwarf, the eclipse would occur at phase $\phi=0.5$ in the radial velocity epehmeris. At $\phi=0.348$ (44), the eclipse indeed occurs early, by $55 \pm 16^{\circ}$ of phase. This is similar to the phase offset seen in other SW Sex stars.

SW Sex stars tend to show absorption features in the $\mathrm{He}_{\mathrm{I}}$ lines that appear at positive velocities around phase 0.3-0.4, drift toward lower velocity, and then disappear sometime after phase 0.5. Figure 10 shows a phase-averaged grayscale representation of our spectra. The phase-dependent absorption is obvious, and behaves exactly as expected for an SW Sex star. The Balmer lines have similar behavior, which is obscured here by the choice of grayscale. The Mg lines around 5170 also have transient absorption, a feature seen in PX And as well (Thorstensen et al. 1991). There is also a pair of weak features around $\lambda \lambda 6348$ and 6371, which we tentatively identify as Si II.
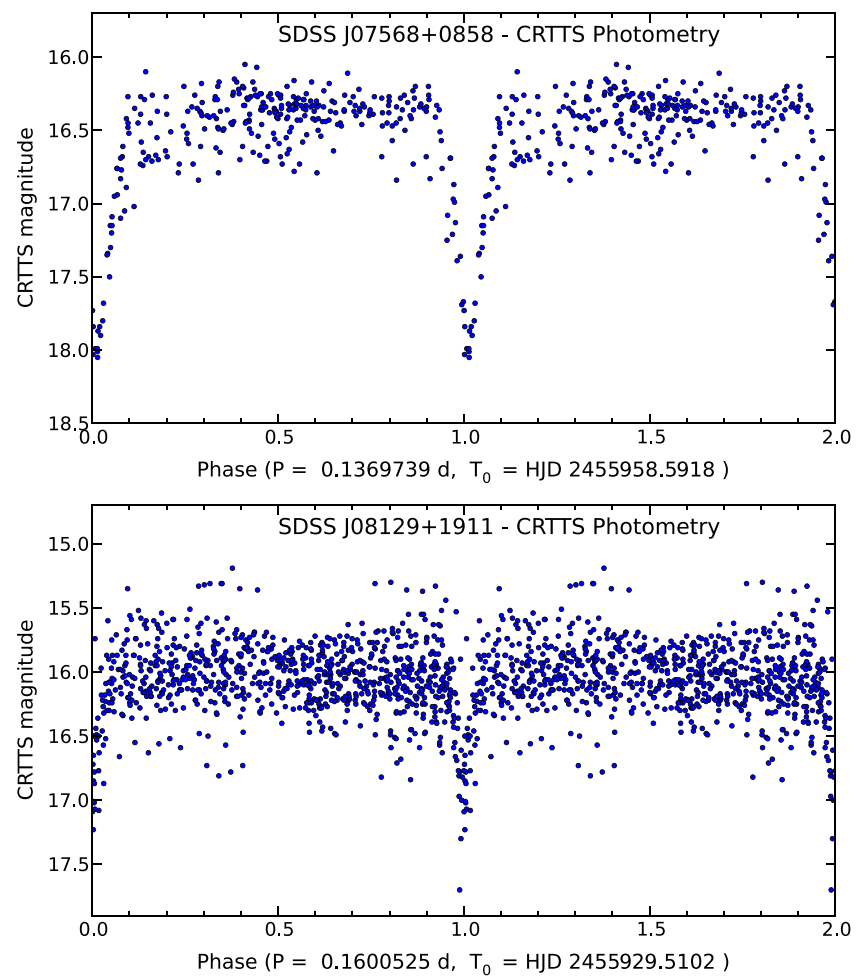

Figure 9. Archival magnitudes of SDSS0756 and SDSS0812 from CRTTS, folded using refined values of the eclipse period.

He II $\lambda 5411$ is weakly visible in emission, without transient absorption.

Rodríguez-Gil et al. (2007) classified this as an SW Sex star without benefit of time-series spectroscopy. The more detailed data presented here resoundingly confirm their classification, as well as refining the orbital period.

\subsection{SDSS J08129+1911}

Gülsecen and Esenog̃lu (2014) present three nights of timeseries photometry of this object that shows eclipses with a period of 0.160046(46) days and a depth of somewhat over 1 mag. Our radial velocity period, 0.1600 (2) days, is consistent with this but somewhat less precise. They also find evidence for a periodicity near 0.148159 days, which they interpret as a possible negative superhump.

The spectrum has a blue continuum and rather modest emission lines- $\mathrm{H} \alpha$ has an equivalent width of about $27 \AA$ and a FWHM $\sim 1100 \mathrm{~km} \mathrm{~s}^{-1}$. The spectrum, and the $3.84 \mathrm{hr}$ orbital period, suggests that this is a novalike variable, or possibly a $\mathrm{Z}$ Cam-type dwarf nova in an extended standstill. Negative superhumps are usually seen in novalike variables in this period range, so the possible negative superhump would tend to favor the novalike interpretation. Also, the CRTSS light curve shows the source steady, with occasional downward excursions in which the object was apparently caught in eclipse (see below), while a Z Cam star would be expected to return to quiescence from time to time. The SDSS spectrum shows only a weak bump near He II $\lambda 4686$, suggesting that this is not a magnetic system. The phase-averaged spectrum (not shown) does not show phase-dependent absorption, suggesting that this is not an SW Sex star.

Because of the eclipse, we examined the CRTTS magnitudes using the procedures described for SDSS J07568+0858. This 

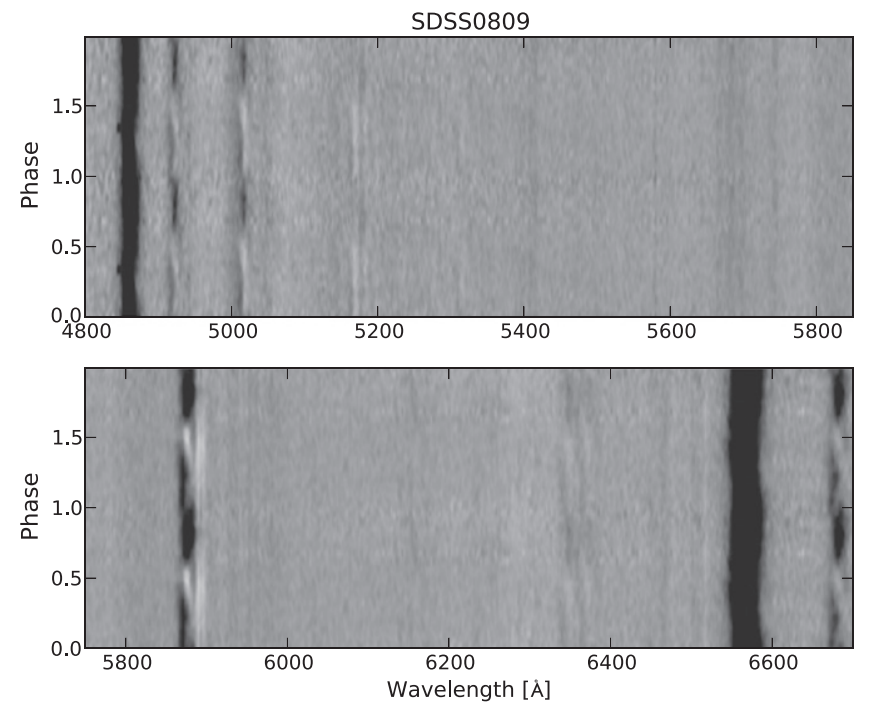

Figure 10. A grayscale representation of the rectified spectra of SDSS0809. Orbital phase increases upward; each line in the image is the average of spectra near that orbital phase, smoothed with a Gaussian over phase. The grayscale is negative (black = bright), from 0.8 to 1.3 times the continuum in order to bring out faint features. The zero of phase is from the eclipse ephemeris from Rodríguez-Gil et al. (2007).

resulted in a refined $P_{\text {orb }}=0.1600525(3)$ days. Figure 9 shows the CRTTS data folded on the refined period.

\subsection{SDSS J08138+2813}

The mean spectrum of SDSS 0813 shows a strong, blue continuum; the emission equivalent width of $\mathrm{H} \alpha$ is around $40 \AA$, and its FWHM is $\sim 950 \mathrm{~km} \mathrm{~s}^{-1}$. Emission at He II $\lambda 4686$ has about half the strength of $\mathrm{H} \beta$. Szkody et al. (2005) found a preliminary $2.7 \mathrm{hr}$ radial velocity period, roughly consistent with our measurement, $P_{\text {orb }}=2.928(9) \mathrm{hr}$. This appears to be a novalike variable just at the long end of the period gap.

As with most of the systems studied here, the flux level in our spectra is consistent with that from the SDSS spectra, and with the SDSS magnitudes. However, in the Digitized Sky Survey red image, taken 1989 November 02, the object is several magnitudes fainter than at other epochs. Like many other novalike variables, this object therefore appears to be a VY Scl star-a cataclysmic that dips into low states (Honeycutt \& Kafka 2004). Consistent with this, the CRTTS light curve, which is not densely sampled, mostly shows slow fading over the past $\sim 8 \mathrm{yr}$ from magnitude 16.5 to $\sim 18$. The phaseaveraged spectrum (not shown) is unremarkable, and in particular does not show phase-dependent absorption.

\subsection{SDSS J08387+4910}

This is an SU UMa-type dwarf nova, for which Kato et al. (2009) give a late superhump period $P_{\text {sh }}=102.92(3)$ minutes. Gänsicke et al. (2009) estimate an orbital period of 99.7 minutes based on the superhump. Szkody et al. (2002) give $g=19.59$, but the CRTTS light curve shows a typical minimum near 18.2. Our synthesized $V$ magnitude is 18.5 , so the SDSS magnitude appears to have been taken during an especially low state. The flux level in our spectrum is also significantly brighter than the SDSS spectrum (Szkody et al. 2002), though the spectral appearance is fairly similar. There are differences; in our spectrum, the emission equivalent width of $\mathrm{H} \alpha$ is $\sim 110 \AA$, about half that in the SDSS spectrum, and its FWHM is $\sim 1600 \mathrm{~km} \mathrm{~s}^{-1}$. $\mathrm{H} \alpha$ is barely double-peaked, suggesting an intermediate orbital inclination.

The radial velocities indicate $P_{\text {orb }}=101.29(0.28)$ minutes, slightly longer than predicted based on the superhump, and yields a superhump period excess

$$
\epsilon=\frac{P_{\mathrm{sh}}-P_{\mathrm{orb}}}{P_{\text {orb }}}=0.016(3) .
$$

Kato et al. (2009) give a relation (their Equation (4)) between the late superhump period (their $P_{2}$ ) and the superhump period excess, which predicts $\epsilon=0.027$ at this period. The superhump period excess $\epsilon$ can be used as a measure of the mass ratio $q=M_{2} / M_{1}$ Patterson (1998). If confirmed, the unexpectedly low $\epsilon$ should indicate an atypically small $q$. The spectroscopic period is based on only two nights of data, though, so a more precise measurement would be especially desirable.

\subsection{SDSS J08440+0239 = V495 Hya}

This is listed as a U Gem-type dwarf nova in the General Catalogue of Variable Stars (Samus et al. 2006), ${ }^{7}$ with an uncertain magnitude of 14 at maximum. The CRTTS light curve shows two outbursts in the five seasons with good coverage.

Of our 92 spectra, 89 are are from 2006 January, and the other three are from 2004 January. The spectra from the two runs appeared similar, but the sparse 2004 January spectra were not used in the analysis.

The mean spectrum shows relatively narrow Balmer lines, with $\mathrm{H} \alpha$ having a FWHM of $600 \mathrm{~km} \mathrm{~s}^{-1}$. He II $\lambda 4686$ is barely detected, and the Fe I line near $\lambda 5169$ is present as well. The continuum is nearly flat, but rises slightly to the red and shows the undulations from a weak M-dwarf contribution. The velocity time series from 2006 January spans $8.7 \mathrm{hr}$ of hour angle, and gives an unambiguous period near $4.97 \mathrm{hr}$, among the longer periods found here.

Using the procedure described in Section 2.3, we estimated the secondary's spectral type to be M2.5 \pm 1.5 subclasses, and its flux contribution to be equivalent to $V=21.5 \pm 0.7$. Repeating the procedure using the archived SDSS spectrum and M-dwarf templates assembled from SDSS data by Bochanski et al. (2007) gave an almost identical result. The secondary's spectral type is close to the that expected at this orbital period (Knigge 2006). The Baraffe \& Kolb (2000) evolutionary calculations indicate a wide range of possible secondary masses at this $P_{\text {orb }}$, so that the secondary's radius is constrained only to $-5 \log _{10}\left(R / R_{\odot}\right)=1.6 \pm 0.4$. In the observed spectral type range, a $1 R_{\odot}$ star would have $M_{V}=8.5 \pm 1.0$. The absolute magnitude of the secondary is therefore $M_{V}=10.1 \pm 1.1$, yielding $m-M=10.9 \pm 1.3$. The Schlegel et al. (1998) dust maps give a small reddening of $E(B-V)=0.04$ at this location $(l=224.08, b=26.13)$, so $(m-M)_{0}=10.8 \pm 1.3$. The best nominal distance is therefore $1400 \mathrm{pc}$, but with almost a factor of two uncertainty in either direction. The calculation above, combined with the observed magnitude, suggests that this system has $M_{V} \sim 8$, consistent with its identification as a dwarf nova.

\footnotetext{
7 Updated versions of the GCVS can be retrieved from http://sai.msu.su/gcvs/ gcvs/iii/html/.
} 


\subsection{SDSS J09127+6209}

This system was discovered by SDSS (Szkody et al. 2011) and apparently independently by Wils et al. (2010), who searched SDSS and other catalogs for CV candidates. In our average spectrum, $\mathrm{H} \alpha$ has an emission equivalent width of nearly $200 \AA$, double-peaked profile with peaks at $\pm 500 \mathrm{~km} \mathrm{~s}^{-1}$, and and a FWHM of $1800 \mathrm{~km} \mathrm{~s}^{-1}$. The double peaks and sizeable radial velocity amplitude $(K=100 \pm$ $15 \mathrm{~km} \mathrm{~s}^{-1}$ ) suggest that the inclination is fairly high, but the system is not known to eclipse. Our spectra, nearly all from 2013 January, do not extend far enough to the red to detect the red dwarf that features prominently in the Wils et al. (2010) spectrum.

Only the 2013 data were extensive enough to analyze for radial velocity. The parameters listed in Table 3 are from the 2013 January data. The period, 115.39(13) minutes, agrees with the $\sim 113$ minutes preliminary period found by Szkody et al. (2009). The spectrum and period are typical of SU UMatype dwarf novae. The CRTTS shows a single possible outburst of $\sim 2 \mathrm{mag}$, but coverage is not heavy. If a future outburst shows superhumps, our period is precise enough to yield an accurate superhump period excess.

\section{9. $S D S S J 09168+2849=H H C n c$}

This dwarf nova shows four outbursts in its CRTTS light curve, which covers nine seasons, so outbursts are fairly infrequent. The spectrum shows a contribution from an M-type companion. We assume this is from the secondary star, but the M-type spectrum is too faint to show radial velocity variation, so we cannot entirely rule out a superposition. The FWHM of the $\mathrm{H} \alpha$ emission is $490 \mathrm{~km} \mathrm{~s}^{-1}$ in our summed spectrum, which is fairly narrow for a CV.

As one might expect from the narrowness of the line, the radial velocity amplitude is low, and most of the time the orbital motion is masked by small fluctuations that are probably due to small changes in the emission-line profiles. Our efforts to find $P_{\text {orb }}$ were stymied on several occasions, but in 2013 February and March the velocities varied smoothly. The period proved to be $P_{\text {orb }}=4.43 \pm 0.01 \mathrm{hr}$, without ambiguity. The velocity data used in Figure 3 are from the 2013 February/ March observing run.

Using the secondary's contribution, we can estimate a distance in the manner outlined in Section 2.3. The M dwarf appears to be in the range M1.5-M4.0. A rather wide range of masses is possible under different evolutionary scenarios; for purposes of computing the M-dwarf radius, we assumed a uniform distribution from 0.15 to 0.50 solar masses. The Monte Carlo simulation gave a distance of $1500 \pm 500 \mathrm{pc}$. At $1500 \mathrm{pc}$ and $b=43^{\circ}$, $\mathrm{HH} \mathrm{Cnc}$ would lie $1.0 \mathrm{kpc}$ from the Galactic plane.

\subsection{SDSS $J 09197+0857=L V C n c$}

Both our mean spectrum and the discovery spectrum (Szkody et al. 2005) show prominent absorption wings in the Balmer lines, which arise from a white-dwarf photosphere; Szkody et al. (2005) also estimated a $\sim 1.4 \mathrm{hr}$ period from radial velocities. Dillon et al. (2008) found a photometric period of $81.6 \pm 1.2$ minutes in a single night of time series photometry. Mukadam et al. (2007) detected a 260 s oscillation in the light curve, which they interpreted as a likely non-radial oscillation, an interpretation corroborated by Woudt et al.
(2012). The radial-velocity period found here, 81.32 (17) minutes or $1.36 \mathrm{hr}$, agrees with and improves on the earlier determinations. The prominent white-dwarf photosphere, very short $P_{\text {orb }}$, and apparent white dwarf oscillations are all reminiscent of GW Lib (Szkody et al. 2000; van Zyl et al. 2004; Thorstensen et al. 2002). The CRTTS shows no clear-cut outbursts despite heavy coverage over $8 \mathrm{yr}$, though the source grew brighter by $\sim 0.5$ mag toward the end of the 2006 season.

The USNO B1.0 catalog (Monet et al. 2003) lists a proper motion of $60 \mathrm{mas} \mathrm{yr}^{-1}$ in a position angle of $262^{\circ}$. The sizeable proper motion, as well as the visibility of the white dwarf, suggest a relatively nearby distance. We therefore began parallax observations (Thorstensen 2003; Thorstensen et al. 2008) of this object in 2006 January, and now have 78 images taken at 12 epochs. This sequence yields a relative parallax $\pi_{\text {rel }}=4.7 \pm 1.7$ mas, and a relative proper motion of 59 mas $\mathrm{yr}^{-1}$ in a position angle of $266^{\circ}$, in excellent agreement with the USNO B1.0. Correcting for the estimated distances of the reference stars gives $\pi_{\mathrm{abs}}=5.5$ mas. The uncertainty estimated from the scatter of the measured parallaxes of similarly faint stars in the field corroborates the uncertainty derived from the goodness of fit, so we adopt $\sigma_{\pi}=1.7$ mas. Since $\pi_{\text {abs }} / \sigma_{\pi}<4$, the classic Lutz-Kelker correction does not formally converge, so following Thorstensen (2003) we adopt a Bayesian approach to the distance estimate, which combines the parallax with prior information ("priors") from the proper motion, and estimated luminosity. We use GW Lib to construct the luminosity prior. Thorstensen (2003) gives a distance of $104(30,-20) \mathrm{pc}$ for this star, and our parallax images give a mean $V=16.96$, while SDSS 0919 has $V=18.30$, which (if it were identical to GW Lib) would put it about $190 \mathrm{pc}$ distant with $M_{V} \sim 11.9$. Taking this as our luminosity prior, assigning it a 1.5 mag uncertainty, then yields a final Bayesian estimate of $195(+54,-39) \mathrm{pc}-$ almost exactly the inverse of the parallax, and also just as expected from scaling the GW Lib distance.

At $195 \mathrm{pc}$, the transverse velocity (corrected to the local standard of rest, or LSR) would be $43 \mathrm{~km} \mathrm{~s}^{-1}$. The velocity fit in Table 3 gives a barycentric systemic velocity of $+73 \mathrm{~km} \mathrm{~s}^{-1}$; taking this at face value, the space velocity relative to the LSR is $U, V, W=+71,-30,+11 \mathrm{~km} \mathrm{~s}^{-1}$. This is a rather large inplane velocity, suggesting that the system belongs to the old disk.

\subsection{SDSS J09224+3307}

The mean spectrum is typical of a dwarf nova at minimum light; $\mathrm{H} \alpha$ has an emission equivalent width of $160 \AA$ and a FWHM of $1300 \mathrm{~km} \mathrm{~s}^{-1}$. He I $\lambda 5876$ is double-peaked, with the peaks separated by $880 \mathrm{~km} \mathrm{~s}^{-1}$, but the double peaks of the Balmer lines are less distinct. The orbital inclination is likely to be unremarkable.

We observed SDSS0922 with the WHT on three nights in 2007 March, under mediocre conditions, and found a $\sim 90$ minutes radial velocity period, but the daily cycle count remained ambiguous. MDM observations taken 2013 February covered a greater range of hour angle and resolved the ambiguity. Table 3 gives the best fits to both data sets and the weighted average period, which amounts to 94.56(12) minutes. The two data sets give significantly different values of the average velocity $\gamma$, but we do not think this discrepancy is likely to be physically meaningful. To construct the 
periodogram and folded velocity curve (Figure 3), we adjusted the data to their weighted average $\gamma$ velocity by subtracting $24 \mathrm{~km} \mathrm{~s}^{-1}$ from the WHT velocities, and adding $33 \mathrm{~km} \mathrm{~s}^{-1}$ to the MDM velocities.

The period and spectrum both suggest that this is a dwarf nova of the SU UMa subtype, but outbursts have not been confirmed. The CRTTS light curve shows variation mostly between 18 and 19 mag, with a few points brighter than 17th mag that may have been weak or poorly covered outbursts.

\subsection{SDSS J09459+2922}

The mean spectrum shows a blue continuum with strong emission lines- $\mathrm{H} \alpha$ is single-peaked, with an emission equivalent width of $180 \AA$ and a FWHM of $1000 \mathrm{~km} \mathrm{~s}^{-1}$. Although the system is relatively faint at $g=19.1$, the $\mathrm{H} \alpha$ emission velocities were well behaved, making the period determination relatively straightforward. We have only 22 velocities from two successive nights, yielding $P_{\text {orb }}=92.0 \pm$ 0.3 minutes. The velocity amplitude $K=255 \pm 22 \mathrm{~km} \mathrm{~s}^{-1}$, which is larger than usually seen in disk systems; in addition, emission line profiles are single-peaked, while an edge-on disk system tends to have doubled lines. This suggests that the system is magnetic, but the $\mathrm{He}$ II $\lambda 4686$ line, which is usually strong in magnetic systems, is quite weak. Time-series polarimetry would be a useful diagnostic.

The CRTS light curve mostly shows variation between 18.5 and 19.5 with occasional points fainter than 20th, and no outbursts.

\subsection{SDSS J10133+4558}

This did not yield a period easily, so we observed it in in 2008 January, 2010 March, and 2011 January. On the last run, we used the $1024^{2}$ CCD "Templeton," which covered a narrower spectral range than the "Echelle" chip used on the other runs. The mean spectrum from the earlier runs gives a synthesized $V=19.0$, but the 2011 January data give $V=19.6$, as a consequence of a fainter continuum. The emission line fluxes did not appear to change significantly.

The number of cycles elapsed between the widely separated observing runs is uncertain. Because of this, the period given in Table 3 is the weighted average derived from separate fits to the 2008 January and 2011 January velocities, which were extensive enough to fit.

The spectrum (Figure 4) is typical of a dwarf nova at minimum light, but the CRTTS light curve shows no outbursts despite 232 epochs of coverage in eight seasons, so outbursts are likely to be infrequent. Infrequent outbursters often resemble WZ Sge, for which all observed outbursts have been superoutbursts (see, e.g., Patterson et al. 2002). It remains to be seen whether this object behaves similarly.

\subsection{SDSS J10197+3357 = HS $1016+3412=A C L M i$}

This object was originally found in the Hamburg Quasar Survey; Aungwerojwit et al. (2006) classified it as a dwarf nova. The CRTTS light curve shows the source mostly near 18th mag, with two outbursts to $\sim 15 \mathrm{mag}$ in $8 \mathrm{yr}$. Aungwerojwit et al. (2006) measured $P_{\mathrm{orb}}=114.3 \pm 2.6$ minutes from emission-line radial velocities. The period we find here, $114.0 \pm 0.2$ minutes, confirms and refines their result. Dwarf novae in this period range are generally SU UMa stars, which show superoutbursts during which they develop superhumps. If this proves to be the case for this object, a usefully precise superhump period excess can be calculated using our improved period.

\subsection{SDSS J10233+4405 = NSV $4838=U M a 8$}

Downes et al. (2001) list this SU UMa-type dwarf nova as "UMa8." Imada et al. (2009) studied two superoutbursts and found a superhump period $P_{\mathrm{sh}}$ near 0.0699 days, or 100.6 minutes; comparing this to our preliminary orbital period, they estimated a mass ratio $q=0.13$.

Our spectrum (Figure 4) is typical of a quiescent dwarf nova. The $\mathrm{H} \alpha$ emission line has an EW of $140 \AA$, two peaks separated by $\sim 900 \mathrm{~km} \mathrm{~s}^{-1}$, and a FWHM $\sim 2100 \mathrm{~km} \mathrm{~s}^{-1}$, suggesting a high orbital inclination. We cannot determine an unambiguous daily cycle count from our radial velocities- the most likely choice is 14.7 cycle day $^{-1}$, but 15.7 and (oddly) 10.4 cycles day $^{-1}$ are possible. However, the single best choice, corresponding to 97.9(8) minutes, is as expected given the superhump period, so the alias choice is secure. Because our data are from several observing runs, with an unknown cycle count between them, we derived the period in Table 3 by fitting the velocities from different runs separately and averaging the results.

\subsection{SDSS $J 10280+2148=1 \mathrm{H} 1025+220=$ Leo5}

This object was apparently discovered because of its X-ray emission, and listed as "Leo5" in the Downes et al. (2001) catalog, which credits the identification to R. Remillard. The spectrum published by Munari et al. (1997), the SDSS spectrum (Szkody et al. 2011), and our averaged spectrum (Figure 4) are all similar, showing a blue continuum with weak Balmer emission lines. Although the spectrum suggests this is a novalike variable, Wils et al. (2011) studied its variation and found it to be a Z Cam star.

Most of our spectra are from 1998 January, but we have data from several other runs up as well. ${ }^{8}$ The radial velocity period, $3.505(4) \mathrm{hr}$, is determined with little ambiguity in the daily cycle count, but the data do not include the number of cycles elapsed between observing runs. The quoted period uncertainty here and in Table 3 is derived from the 1998 January data alone. The period is typical for a $\mathrm{Z}$ Cam star.

\subsection{SDSS J11313+4322 = MR UMa}

This CV was discovered by Wei et al. (1997) as the optical counterpart of a ROSAT X-ray source. It is an SU UMa star, for which Kato et al. (2009) list a superhump period near 0.0652 days in the main part of the outburst. Szkody et al. (2006) list $g=16.17$, but our spectrum indicates $V=17.9$, so the star was evidently above minimum when the SDSS direct image was taken. The continuum level in the SDSS spectrum (in Figure 1 of Szkody et al. 2006) is nearly identical to ours, so our spectra were apparently both taken in quiescence.

\subsection{SDSS J11322+6249}

The mean spectrum shows a strong, blue continuum and single-peaked emission lines. The $\mathrm{H} \alpha$ emission line has an equivalent width of $85 \AA$ and a FWHM of $800 \mathrm{~km} \mathrm{~s}^{-1}$. H $\beta$ is stronger than $\mathrm{H} \alpha$, with a flux ratio $F(\mathrm{H} \beta) / F(\mathrm{H} \alpha) \sim 1.7$. He II

\footnotetext{
8 The data were published in author CJT's PhD thesis (Taylor 1999), but have not been published in a journal article until now.
} 
$\lambda 4686$ is present at $\sim 1 / 7$ the strength of $\mathrm{H} \beta$. We took spectra of SDSS1132 on two observing runs, in 2008 January and March. The January data indicated a period near 99 minutes, and the March data resolved ambiguities in the daily cycle count. The number of cycles that elapsed in the interval between the two runs is unknown, but the set of possible periods can be expressed as

$$
P=\frac{45.228 \pm 0.004 \text { days }}{656 \pm 7},
$$

where the denominator is an integer.

The subclassification of this object is ambiguous. The continuum and $\mathrm{He}$ II line suggests that this might be an AM Her star (or polar), but the line profiles remain symmetrical around the orbit, while AM Her stars typically show highly variable, asymmetric profiles from the accretion column (see, e.g., Schwope et al. 1999). The object is listed in the ROSAT faint source catalog (Voges et al. 2000). Dwarf nova outbursts do not appear to have been detected; the CRTTS light curve shows erratic variation between magnitude 17.5 and 19.5 in their system. This may be a novalike variable with a period shortward of the gap, which if confirmed would be unusual.

\subsection{SDSS J12442+3004}

This dwarf nova was discovered by the CRTTS prior to its listing by SDSS; it is also listed in the GALEX catalog and was selected as a QSO candidate by Atlee \& Gould (2007). The mean spectrum shows the strong emission lines typical of short-period dwarf novae at minimum light. We have spectra from two observing runs, one in 2009 March and the other in 2010 March. The 111.5 minutes period is found from an unambiguous daily cycle count but the number of cycles elapsed between observing runs is unknown; the exact period is constrained to

$$
P_{\text {orb }}=\frac{382.824 \pm 0.002 \text { days }}{4944 \pm 25},
$$

where the denominator is an integer. We are unaware of any detection of superhumps, but at this orbital period they are likely to occur.

\subsection{SDSS J14299+4145}

The SDSS spectrum (Szkody et al. 2005) shows a blue continuum with single-peaked emission lines, and with He II $\lambda 4686$ about half the strength of $\mathrm{H} \beta$. The Balmer decrement is inverted, with $\mathrm{H} \beta$ much stronger than $\mathrm{H} \alpha$. Our mean spectrum is similar. We have data from 2005 March and 2005 June; in March, our spectra implied $V=18.2$, but in June it had faded to $V=19.2$. The object appears much fainter on all the digitized Schmidt plate survey images than it does in SDSS, so it is evidently highly variable. The CRTTS data are very sparse in this location, but show variation between magnitude 18 and 21.

Our $\mathrm{H} \alpha$ radial velocities favor a period near 99 minutes, or 14.61 cycle day $^{-1}$, but daily cycle-count aliases near 15.68 and 13.57 cycle day $^{-1}$ are also possible. The number of cycles elapsed in the 100 days interval between our 2005 March and June runs is also not determined. Our period corroborates the $\sim 1.4 \mathrm{hr}$ candidate period found by Szkody et al. (2005) in $2.5 \mathrm{hr}$ of spectroscopy, but neither determination is definitive.

If the candidate spectroscopic period is $P_{\text {orb }}$, as is nearly always the case, then $P_{\text {orb }}$ is unusually short given the star's other characteristics. The emissions at He II $\lambda 4686$ is about half the strength of $\mathrm{H} \beta$, which is typical for intermediate polars (IPs, or DQ Her stars) - systems containing a magnetic white dwarf that rotates more quickly than the binary orbital frequency. Most IPs, however, have longer orbital periods than this. Polars, or AM Her stars-systems in which a magnetic white dwarf rotates synchronously-often have periods as short as SDSS1429, and can also spend long intervals in very low states, as this object evidently does. However, most polars have $\lambda 4686$ roughly equal to that of $\mathrm{H} \beta$, and the velocity amplitude tends to be very large, because of the high infall velocities in the accretion funnel close to the white dwarf. One of the spectra obtained by Szkody et al. (2005) showed double-peaked Balmer lines and He II $\lambda 4686$ approximately equal to $\mathrm{H} \beta$. The double-peaked lines might be due to transient absorption, which would be reminiscent of an SW Sex star, but as Szkody et al. (2005) remark, SW Sex phenomena tend to be found in objects with $P_{\text {orb }}>3 \mathrm{hr}$.

The nature of this object therefore remains rather problematical. If it is a magnetic system, time-series photometry might reveal a white dwarf pulsation period.

\subsection{SDSS J15046+0847 = "Bool"}

Filippenko et al. (1985) discovered this object on an objective-prism plate. The spectrum appears typical for a dwarf nova at minimum light, but outbursts do not appear to have been recorded; in particular, the CRTTS shows no outbursts despite very good coverage. The emission lines are single-peaked, and the FWHM of $\mathrm{H} \alpha$ is $770 \mathrm{~km} \mathrm{~s}^{-1}$, suggesting a fairly low orbital inclination. Our spectra, from three successive nights in 2010 March, span only $5.3 \mathrm{hr}$ of hour angle, but the radial velocity period is determined to be 116.5 (3) minutes without significant ambiguity. This period is typical of SU UMa-type dwarf novae, so superhumps are likely in the event of an outburst.

\subsection{SDSS J15365+3328}

This object was discovered independently by the CRTTS as a dwarf nova. The CRTTS light curve shows four outbursts, reaching an apparent magnitude $m \sim 15$, with a minimum typically $m \sim 18.5$, with some fainter interludes.

The mean spectrum is typical of a dwarf nova at minimum light, with emission lines of $\mathrm{H}, \mathrm{He}$, and Fe II $\lambda 5169$. The lines are slightly double-peaked, with the red and blue peaks of $\mathrm{H} \alpha$ separated by $\sim 600 \mathrm{~km} \mathrm{~s}^{-1}$. The $\mathrm{H} \alpha$ line has an emission equivalent width near $100 \AA$ and a FWHM of $\sim 1500 \mathrm{~km} \mathrm{~s}^{-1}$. The period, 132.7(3) minutes, is unusual for being near the lower edge of the gap, but it appears to be reliable; Monte Carlo tests give a probability of less than $1 \%$ for a cycle count error. At this $P_{\text {orb }}$, the superhumps and superoutbursts characteristic of an SU UMa star should occur.

$$
\text { 3.23. SDSS J15567-0009 = V493 Ser }
$$

Woudt et al. (2004) reported photometry and found a period of $0.07408(1)$ days; their choice of daily cycle count was, however, not entirely secure. Kato et al. (2009) observed a superoutburst in 2007 that showed a mean superhump period of $0.082853(5)$ days. Because superhump periods are almost always only a few per cent longer than orbital periods, they concluded that the Woudt et al. (2004) period is the 1 day alias of the correct period, which would then be near 0.08001(1) days. 
Our radial-velocity period supports this revision. At the time of our observations (2003 June 20-24), the source transited well before midnight, so we obtained only a $5.3 \mathrm{hr}$ span of hour angle. Even so, the Monte Carlo test (Thorstensen \& Freed 1985) gives a discriminatory power greater than 0.9 , and a correctness likelihood near unity for our alias choice. The radialvelocity period is 0.08012 (8) days - consistent, within the errors, with that inferred by Kato et al. (2009). The alias choice is therefore secure, and the Woudt et al. (2004) period is superseded.

\subsection{SDSS $J 16101+0352=V 519 \mathrm{Ser}$}

This object was discovered as the counterpart of a ROSAT Xray source (Jiang et al. 2000). Rodrigues et al. (2006) detected strong circular polarization, confirming it as a polar, or AM Her star. From their photometry and polarization they found $P_{\text {orb }}=0.13232(4)$ days.

Our radial velocities span the interval from 2000 April to 2011 June. An unambiguous cycle count over that interval yields $P_{\text {orb }}=0.1322739$ (2) days, consistent with the Rodrigues et al. (2006) result but more precise because of the long time base.

The discovery spectrum shows a clear contribution from an M-dwarf secondary star, which Jiang et al. (2000) classify as M4 or M5. This is not evident in SDSS spectrum (Szkody et al. 2009), which has $\sim 8$ times the flux, obscuring the secondary's contribution. Our mean spectrum (Figure 6) shows the source somewhat brighter than the discovery spectrum, and the secondary is detected.

To classify the secondary, we averaged together the spectra with the lowest continuum levels (fainter than synthesized $V=18.3$ ), and compared this average against a library of spectra of M dwarfs taken with the same instrument. Types M4 through M5 did appear to match somewhat better than earlier types. An argument similar to that used in SDSS J08055+0720 — using the secondary's inferred surface brightness and the constraints on its radius given the orbital period — gives a distance estimate of $450(+120,-100)$ pc for this system.

\subsection{SDSS J16191+1351}

The CRTTS light curve of this object shows irregular variations between $m=17$ and 18 magnitude, punctuated by a large number of short outbursts to $m \sim 15$, marking this as a frequently outbursting dwarf nova. Indeed, the source was in outburst during follow-up spectroscopy reported by Szkody et al. (2009).

The continuum in our spectrum sweeps upward toward the blue, suggesting that the object was not completely at minimum light. The synthetic $V=17.24$ corroborates this conclusion. Even so, a weak contribution from a secondary star is discernible, in addition to the emission lines typical of dwarf novae. Using the procedures described in Section 2.3, we classify the secondary as $\mathrm{K} 4 \pm 2$ subclasses, and estimate a distance of $3100(+800,-700)$ pc. The Galactic latitude of nearly $40^{\circ}$, so our distance puts the system about $2 \mathrm{kpc}$ away from the Galactic plane.

Both the emission and absorption line velocities show orbital modulation at the same period (within the uncertainties). The difference in the phase of the emission and absorption modulations is $0.478(16)$ cycles, consistent with the value of
0.5 expected if the emission traces the motion of the white dwarf. ${ }^{9}$ For plausible masses, the velocity amplitude $K$ suggest an intermediate orbital inclination. If we assume that (1) the emission and absorption $K$-velocities faithfully represent with the white dwarf and secondary motions, respectively, and (2) the white dwarf has $0.7 M_{\odot}$, then the nominal $K_{2} / K_{1}$ gives 0.56 $M_{\odot}$ for the secondary, and the inclination is around $40^{\circ}$. Note that we have not measured this-the calculation is simply to show that the data are consistent with ordinary values, and that the system is rather unlikely to eclipse.

\subsection{SDSS J16539+2010 = V1227 Her}

This is an SU UMa-type dwarf nova. When Szkody et al. (2006) performed follow-up photometry on this object, they found it in outburst and discovered a $1.58 \mathrm{hr}$ superhump modulation, implying an orbital period near 91 minutes. Kato et al. (2010) report $P_{\mathrm{sh}}=93.54(4)$ minutes early in the outburst, increasing to 93.91(4) minutes later. Our spectroscopic orbital period is 90.86(13) minutes, so that $\epsilon=\left(P_{\mathrm{sh}}-P_{\text {orb }}\right) / P_{\text {orb }}$ evolves from 0.029 to 0.034 during the outburst. This is approximately as expected at this orbital period (e.g., Equation (2) of Gänsicke et al. 2009).

\subsection{SDSS J16569 + $2121=V 1229 \mathrm{Her}$}

Our spectra are all from 2006 June. The mean spectrum (Figure 7) shows broad emission lines with incipient double peaks; $\mathrm{H} \alpha$ has a FWHM of over $1700 \mathrm{~km} \mathrm{~s}^{-1}$ and an equivalent width $\sim 130 \AA$ A. Judging from the breadth of the lines, the orbital inclination is likely to be high. However, Southworth et al. (2007) did not detected any eclipses in a photometric time series longer than the 96.9 minutes orbital period found here. The period and spectrum suggest that this is an SU UMa-type dwarf nova; if superhumps and superoutbursts are detected, this would be confirmed. The CRTTS light curve shows no outbursts, despite rather dense sampling, so outbursts appear to be infrequent.

\subsection{SDSS J16598+1927}

The mean spectrum (Figure 7) shows a strong, blue continuum and relatively weak lines - the equivalent width of $\mathrm{H} \alpha$ is only $18 \AA$. The He I lines are barely detected at this signal-to-noise ratio, but He II $\lambda 4686$ is present. There is sharp absorption at the $\mathrm{NaD}$ lines, which is very likely interstellar.

Southworth et al. (2008) obtained a short spectroscopic time series, which failed to show an orbital period. Our velocities were noisy-we needed 106 velocities spread over five different observing runs to secure the daily cycle count, so their non-detection of $P_{\text {orb }}$ is not surprising. The periodogram of our velocities shows much fine-scale structure near the orbital frequency $\left(7.07\right.$ cycle day $^{-1}$, or $P_{\text {orb }} \sim 0.141$ days).

The spectrum and orbital period of this object are consistent with a novalike variable, though it could also be a Z Cam star in an extended high state. The CRTTS light curve shows only irregular variations between 16.5 and $17.5 \mathrm{mag}$, which suggests that this is a novalike.

\footnotetext{
The mean velocities $\gamma$ of the absorption and emission-79(7) and 19 (8) $\mathrm{km} \mathrm{s}^{-1}$ respectively - are formally very different, but the emission velocities especially can be skewed by line-profile effects, so we are reluctant to ascribe physical significance to this.
} 


\subsection{SDSS J17301+6247}

This is an SU UMa-type dwarf nova, with a $P_{\text {sh }}$ near 0.0794 days, or 114.3 minutes (Kato et al. 2009). The emission lines (Figure 8) are single-peaked, suggesting a fairly low orbital inclination. Combining our measured orbital period, 110.3(2) minutes, with $P_{\text {sh }}$ gives a fractional superhump period excess $\epsilon=\left(P_{\mathrm{sh}}-P_{\text {orb }}\right) / P_{\text {orb }}=0.037$, which is close to the expected value for this $P_{\text {orb }}$.

\section{DISCUSSION}

As Gänsicke et al. (2009) emphasize, the SDSS has brought us significantly closer to a true accounting of the $\mathrm{CV}$ period distribution; in particular, over $30 \%$ of their sample of $92 \mathrm{CVs}$ discovered in the SDSS had periods lying in a "spike" in the distribution, which they defined as being from roughly 80 to 86 minutes. The shortest-period object in the present sample is LV Cnc (= SDSS J09197+0857), at 81.3 minutes; the next shortest is V1227 Her (=SDSS J16539+2010), at 90.9 minutes. LV Cnc is therefore the only object in the "spike." The white dwarf in LV Cnc is readily apparent in the spectrum, which marks it as one of the least luminous objects in the sample. Evidently, the present sample cannot be taken as representative, because it is skewed toward higher luminosities. This is not surprising, since spectroscopy of fainter targets can be very difficult. It is fortunate, then, that many of the shortest-period CVs undergo superoutbusts and show superhumps, which can be used to derive approximate periods that are good enough for many purposes (see, e.g.. Kato et al. 2009, 2010).

In general, longer-period systems tend to be more luminous (Patterson 1984), and hence more amenable to spectroscopy. Indeed, 11 of the 29 systems discussed here have periods longer than $3 \mathrm{hr}$-in order of increasing period, they are SDSS0813, 1610, 0809, 0756, 1659, 1028, 0812, 0916, 0844, 0805, and 1619. As noted above, it is relatively easy to find rough orbital periods for short-period dwarf novae that show superoutbursts. Spectroscopic studies such as the present one are needed in part to ensure that the distribution of known $\mathrm{CV}$ periods does not become skewed toward the short end.

The number of known and suspected CVs has exploded in recent years because of deep, all-sky variability surveys such as the CRTTS, ASAS-SN (Shappee et al. 2014), and MASTER (Lipunov et al. 2010). However, because of the enormous number of variable stars in the sky, only objects with largeamplitude variability tend to be flagged as CVs; in the CRTTS, for example, only objects with $\Delta m>2 \mathrm{mag}$ are flagged as CV candidates (Drake et al. 2009). The sample of CRTTS CVs is consequently biased toward large outburst amplitudes (Thorstensen \& Skinner 2012). Intriguingly, though, Breedt et al. (2014), reviewing more than 1000 CRTTS dwarf nova discoveries over the past $8 \mathrm{yr}$, find that the rate at which CRTTS discovers frequently outbursting objects has slowed dramatically, while the rate at which it is finding objects that show no previous outbursts has not slowed significantly. This suggests that there is a sizable population of quiescent dwarf novae - or, more properly, dwarf novae with very long outburst recurrence times - waiting to be discovered.

The SDSS CV sample, which was selected by color and spectrum, supports this idea. Seven of the objects in the present sample (SDSS0805, 0919, 0945, 1013, 1132, 1504, and 1656) have spectra consistent with dwarf novae at minimum light, but without known outbursts, even though some have been covered extensively by CRTTS. Variability surveys have also tended to miss other classes of CVs that are not dwarf novae- the socalled novalike variables. The present sample has some seven of these, namely SDSS0756, 0809, 0812, 0813, 1028 (which may be a Z Cam-type dwarf nova), 1429 , and $1659 .{ }^{10}$

It's likely, then, that as new CVs are discovered, they will mostly be (a) dwarf novae that seldom outburst — possibly with arbitrarily long recurrence times-and (b) various kinds of novalike variables. Since essentially all cataclysmic binaries that actively transfer matter are variable stars, these might be found by looking for lower-amplitude variables in surveys such as CRTTS, and sifting these for unusual colors. In the more distant future, a careful analysis of the Large Synoptic Survey Telescope $^{11}$ data should find essentially every $\mathrm{CV}$ in the southern sky to an impressive magnitude limit.

For convenience, we list here some noteworthy results on individual objects:

1. SDSS J07568+0858 and SDSS J08129+1911 were both previously known to be eclipsing novalike variables; we refined their eclipse periods using archival data from CRTTS. This technique may be of interest to other workers in the field.

2. We improve the period of SDSS J08091+3814, and use phase-averaged spectroscopy to corroborate its suggested membership in the SW Sextantis class (Rodríguez-Gil et al. 2007).

3. We measure a parallax-based distance of $195(+54,-39)$ pc for the shortest-period object in our sample, LV Cnc (SDSS J09197+0857). This system appears to be a neartwin of the seldom-outbursting dwarf nova GW Lib.

4. SDSS J09459+2922 has some characteristics of a magnetic $\mathrm{CV}$, and may be a rewarding target for time series polarimetry.

5. The subtype of SDSS J11324+6249 is ambiguous; it may be a novalike variable with an atypically short period.

We gratefully acknowledge support from NSF grants AST0307413, AST-0708810, and AST-1008217. We thank the MDM staff for observing assistance, and Sébastien Lépine for taking some of the parallax data on SDSS 0919. The research leading to these results has received funding from the European Research Council under the EU 7th Framework Programme (FP/2007-20013/ERC Grant Agreement n. 320964 (WDTracer). This paper uses data from the Catalina Sky Survey and Catalina Real Time Survey; the CSS is funded by the National Aeronautics and Space Administration under Grant No. NNG05GF22G issued through the Science Mission Directorate Near-Earth Objects Observations Program. The CRTS survey is supported by the US National Science Foundation under grants AST-0909182.

\section{REFERENCES}

Atlee, D. W., \& Gould, A. 2007, ApJ, 664, 53

Aungwerojwit, A., Gänsicke, B. T., Rodríguez-Gil, P., et al. 2006, A\&A, 455,659

Augusteijn, T., van der Hooft, F., de Jong, J. A., \& van Paradijs, J. 1996, A\&A, 311,889

Baraffe, I., \& Kolb, U. 2000, MNRAS, 318, 354

\footnotetext{
${ }^{10}$ Several of these were discovered by other surveys, but SDSS did select them by color and obtain spectra.

11 An up-to-date description of the LSST project can be found at http://arxiv. org/abs/0805.2366.
} 
Barker, J., \& Kolb, U. 2003, MNRAS, 340, 623

Bessell, M. S. 1990, PASP, 102, 1181

Beuermann, K., Baraffe, I., \& Hauschildt, P. 1999, A\&A, 348, 524

Bochanski, J. J., West, A. A., Hawley, S. L., \& Covey, K. R. 2007, AJ, 133,531

Boeshaar, P. 1976, PhD thesis, The Ohio State Univ.

Breedt, E., Gänsicke, B. T., Drake, A. J., et al. 2014, MNRAS, 443, 3174

Dillon, M., Gänsicke, B. T., Aungwerojwit, A., et al. 2008, MNRAS, 386, 1568

Downes, R. A., Webbink, R. F., Shara, M. M., et al. 2001, PASP, 113, 764

Drake, A. J., Djorgovski, S. G., Mahabal, A., et al. 2009, ApJ, 696, 870

Dworetsky, M. M. 1983, MNRAS, 203, 917

Filippenko, A. V. 1982, PASP, 94, 715

Filippenko, A. V., Sargent, W. L. W., \& Hazard, C. 1985, PASP, 97, 41

Gänsicke, B. T., Dillon, M., Southworth, J., et al. 2009, MNRAS, 397, 2170

Gülsecen, H., \& Esenog̃lu, H. 2014, New A, 28, 49

Horne, K. 1986, PASP, 98, 609

Honeycutt, R. K., \& Kafka, S. 2004, AJ, 128, 1279

Imada, A., Yasuda, T., Omodaka, T., et al. 2009, PASJ, 61, 535

Jiang, X. J., Engels, D., Wei, J. Y., Tesch, F., \& Hu, J. Y. 2000, A\&A, 362,263

Kato, T., Imada, A., Uemura, M., et al. 2009, PASJ, 61, 395

Kato, T., Maehara, H., Uemura, M., et al. 2010, PASJ, 62, 1525

Keenan, P. C., \& McNeil, R. C. 1989, ApJS, 71, 245

Knigge, C. 2006, MNRAS, 373, 484

Kurtz, M. J., \& Mink, D. J. 1998, PASP, 110, 934

Linnell, A. P., Hoard, D. W., Szkody, P., et al. 2007, ApJ, 654, 1036

Lipunov, V., Kornilov, V., Gorbovskoy, E., et al. 2010, AdAst, 2010, 349171

Littlefair, S. P., Dhillon, V. S., Marsh, T. R., et al. 2007, MNRAS, 381, 827

Monet, D. G., Levine, S. E., Canzian, B., et al. 2003, AJ, 125, 984

Mukadam, A. S., Gänsicke, B. T., Szkody, P., et al. 2007, ApJ, 667, 433

Munari, U., Zwitter, T., \& Bragaglia, A. 1997, A\&AS, 122, 495

Patterson, J. 1984, ApJS, 54, 443

Patterson, J. 1998, PASP, 110, 1132

Patterson, J., Masi, G., Richmond, M. W., et al. 2002, PASP, 114, 721

Patterson, J., Thorstensen, J. R., \& Knigge, C. 2008, PASP, 120, 510

Rodrigues, C. V., Jablonski, F. J., D’Amico, F., et al. 2006, MNRAS, 369,1972

Rodríguez-Gil, P., Gänsicke, B. T., Hagen, H.-J., et al. 2007, MNRAS, 377,1747

Samus, N. N., Durlevich, O. V., Zharova, A. V., et al. 2006, AstL, 32, 263

Samus, N. N., Durlevich, O. V., Zharova, A. V., et al. 2012, General Catalogue of Variable Stars Database(Institute of Astronomy of Russian Academy of Sciences and Sternberg State Astronomical Institute of the Moscow State University)
Schlegel, D. J., Finkbeiner, D. P., \& Davis, M. 1998, ApJ, 500, 525

Schneider, D., \& Young, P. 1980, ApJ, 238, 946

Schwope, A. D., Schwarz, R., Staude, A., et al. 1999, in ASP Conf. Ser. 157, Annapolis Workshop on Magnetic Cataclysmic Variables, ed. C. Hellier, \& K. Mukai (San Francisco, CA: ASP), 71

Shappee, B. J., Prieto, J. L., Grupe, D., et al. 2014, ApJ, 788, 48

Southworth, J., Marsh, T. R., Gänsicke, B. T., et al. 2007, MNRAS, 382, 1145

Southworth, J., Gänsicke, B. T., Marsh, T. R., et al. 2008, MNRAS, 391, 591

Szkody, P., Anderson, S. F., Agüeros, M., et al. 2002, AJ, 123, 430

Szkody, P., Anderson, S. F., Brooks, K., et al. 2011, AJ, 142, 181

Szkody, P., Anderson, S. F., Hayden, M., et al. 2009, AJ, 137, 4011

Szkody, P., Desai, V., \& Hoard, D. W. 2000, AJ, 119, 365

Szkody, P., Fraser, O., Silvestri, N., et al. 2003, AJ, 126, 1499

Szkody, P., Henden, A., Agüeros, M., et al. 2006, AJ, 131, 973

Szkody, P., Henden, A., Fraser, O., et al. 2004, AJ, 128, 1882

Szkody, P., Henden, A., Fraser, O. J., et al. 2005, AJ, 129, 2386

Szkody, P., Henden, A., Mannikko, L., et al. 2007, AJ, 134, 185

Taylor, C. J. 1999, PhD thesis, Dartmouth College

Thorstensen, J. R. 2003, AJ, 126, 3017

Thorstensen, J. R., Fenton, W. H., Patterson, J. O., et al. 2002, ApJL, 567, L49

Thorstensen, J. R., Lépine, S., \& Shara, M. 2008, AJ, 136, 2107

Thorstensen, J. R., Patterson, J., Kemp, J., \& Vennes, S. 2002, PASP, 114,1108

Thorstensen, J. R., Patterson, J., Thomas, G., \& Shambrook, A. 1996, PASP, 108,73

Thorstensen, J. R., Ringwald, F. A., Wade, R. A., Schmidt, G. D., \& Norsworthy, J. E. 1991, AJ, 102, 272

Thorstensen, J. R., \& Freed, I. W. 1985, AJ, 90, 2082

Thorstensen, J. R., \& Skinner, J. N. 2012, AJ, 144, 81

Tonry, J., \& Davis, M. 1979, AJ, 84, 1511

Tovmassian, G., Hernandez, M. S., González-Buitrago, D., Zharikov, S., \& García-Díaz, M. T. 2014, AJ, 147, 68

van Zyl, L., Warner, B., O'Donoghue, D., et al. 2004, MNRAS, 350, 307

Voges, W., Aschenbach, B., Boller, T., et al. 2000, IAU Circ., 7432, 1

Warner, B. 1995, Cataclysmic Variable Stars (New York: Cambridge Univ. Press)

Wei, J.-Y., Cao, L., Xu, D.-W., Hu, J.-Y., \& Li, Q.-B. 1997, ChA\&A, 21,146

Wils, P., Gänsicke, B. T., Drake, A. J., \& Southworth, J. 2010, MNRAS, 402, 436

Wils, P., Krajci, T., \& Simonsen, M. 2011, JAVSO, 39, 77

Woudt, P. A., Warner, B., de Budé, D., et al. 2012, MNRAS, 421, 2414

Woudt, P. A., Warner, B., \& Pretorius, M. L. 2004, MNRAS, 351, 1015 